\title{
Solute transport along a single fracture in a porous rock: a simple analytical solution and its extension for modeling velocity dispersion
}

\author{
Longcheng Liu $^{1}$ - Ivars Neretnieks ${ }^{1} \cdot$ Pirouz Shahkarami $^{1}$ (D) S Shuo Meng ${ }^{1}$. \\ Luis Moreno ${ }^{1}$
}

Received: 3 February 2017 / Accepted: 16 June 2017 / Published online: 26 July 2017

(C) The Author(s) 2017. This article is an open access publication

\begin{abstract}
A simple and robust solution is developed for the problem of solute transport along a single fracture in a porous rock. The solution is referred to as the solution to the singleflow-path model and takes the form of a convolution of two functions. The first function is the probability density function of residence-time distribution of a conservative solute in the fracture-only system as if the rock matrix is impermeable. The second function is the response of the fracture-matrix system to the input source when Fickian-type dispersion is completely neglected; thus, the effects of Fickian-type dispersion and matrix diffusion have been decoupled. It is also found that the solution can be understood in a way in line with the concept of velocity dispersion in fractured rocks. The solution is therefore extended into more general cases to also account for velocity variation between the channels. This leads to a development of the multi-channel model followed by detailed statistical descriptions of channel properties and sensitivity analysis of the model upon changes in the model key parameters. The simulation results obtained by the multi-channel model in this study fairly well agree with what is often observed in field experiments - i.e. the unchanged Peclet number with distance, which cannot be predicted by the classical advectiondispersion equation. In light of the findings from the aforementioned analysis, it is suggested that forced-gradient experiments can result in considerably different estimates of dispersivity compared to what can be found in naturalgradient systems for typical channel widths.
\end{abstract}

Pirouz Shahkarami

pirouzs@kth.se

1 Department of Chemical Engineering, Royal Institute of Technology, Stockholm, Sweden
Keywords Fractured rocks - Velocity dispersion · Mathematical model · Matrix diffusion · Taylor dispersion

\section{Introduction and scope}

To aid safety assessment of geological radioactive waste repositories, a variety of models are developed based on the advection-dispersion equation, $\mathrm{ADE}$, to address the problem of water flow and solute transport in fractured crystalline rocks (Berkowitz 2002). The classical ADE models solute transport as if the hydrodynamic dispersion, in its widest sense, behaves similar to a Fickian-diffusion process and it can be characterized by a constant dispersion coefficient, $D_{\mathrm{f}}$, that can be significantly larger than the molecular diffusion coefficient if, for example, Taylor-like dispersion contributes (Taylor 1953).

However, it has been increasingly recognized that the residence time distribution of a solute in fractured rocks cannot be described as a Fickian process (embodied in the ADE) (Matheron and De Marsily 1980). In a survey study, Gelhar et al. (1992) compiled dispersivity observations from 59 different sites and concluded that the dispersion coefficient increases with observation distance which itself is typical of non-Fickian behavior and invalidates the use of the ADE for interpretation of field tracer tests. In an illustrative study, Becker and Shapiro (2000) showed that the classical ADE cannot readily explain the early arrival and late-time behavior of tracer test breakthrough curves, BTCs. The former is of crucial importance for safety assessment of the geological repositories (Rasmuson and Neretnieks 1986). There are also numerous studies indicating that the ADE does not adequately represent the real nature of solute transport in geological formations, which often exhibit anomalous behavior. (Long et al. 1982; Berkowitz et al. 1988; McKay et al. 1993). 
Due to these shortcomings in explaining the observed nonFickian features in solute migration patterns, alternative transport models are proposed to capture the effects of spatial heterogeneities in fractured rocks. These models include the multi-rate mass transfer models (Haggerty and Gorelick 1995; Mahmoudzadeh et al. 2013), the multi-continuum approaches (Šimůnek et al. 2003; Šimůnek and van Genuchten 2008), the continuous time random walk approach (Berkowitz et al. 2006), the time-domain random walk approach (Bodin 2015; Shahkarami et al. 2016), the fractional advection-dispersion equation approach (Benson et al. 2000), as well as the stochastic-convective transport approach (Cirpka and Kitanidis 2000; Ginn 2001).

In this paper, attention is focused on the dispersion effect that may be caused due to the separation of advective transport paths in fractures. The authors propose the multi-channel model to explain the non-Fickian behavior of solute transport in heterogeneous fractured rocks. The idea of the multichannel model essentially stems from the real nature of solute transport in fractured rock. In a real fracture, apertures vary from fully closed to usually less than $1 \mathrm{~mm}$. Some open parts in the fracture are connected and, in the presence of a hydraulic gradient, water will flow with different velocities though the pathways in the fracture. Such a conduit in the fracture is here called a channel. Provided there is a large velocity distribution, the channels may become independent with negligible mass exchange between the neighboring channels (Moreno and Tsang 1994). In this way, spreading of a tracer observed at a downstream location is caused by the solutes having traveled in different channels with different residence times toward the observation point. In literature, this process is often referred to as flow channeling or velocity dispersion (Neretnieks 1983; Becker and Shapiro 2003). In a single channel, the tracer may experience different retarding mechanisms, e.g. matrix diffusion and sorption; thus, the multi-channel model can also describe the consequences of collecting and mixing the solutes advected from different channels with different flow rates and residence times, which have been subject to different spreading mechanisms along the flow paths. The net effect on solute transport is, however, quite different from a Fickian-type dispersion and cannot readily be included in the ADE with a constant dispersion coefficient, because it extrapolates differently with changing travel distance.

The building block of the multi-channel model is a single fracture (or channel) surrounded by a porous rock matrix. A large body of literature has addressed the problem of solute transport in a single-fracture-matrix system and has developed, based on the ADE, analytical solutions that predict the solute concentration along the fracture (Tang et al. 1981; Sudicky and Frind 1982; Hodgkinson and Maul 1988). For the purpose of understanding the consequences of the flow channeling, firstly, this study starts by revisiting the twoparallel-plates model that accounts for the effects of
advection-Fickian-type dispersion and matrix diffusion on solute transport in a single-fracture-matrix system. A much simpler solution is derived compared to that presented by Tang et al. (1981). In the new solution, here referred to as the solution to the single-flow-path model, the effects of dispersion and matrix diffusion on solute transport have been decoupled in the sense that one may consider these two transport mechanisms separately and independently. It is shown that the new solution can be visualized as if the spreading is the result of solutes having fictitiously followed streamlines with different water velocities, provided there is a Fickiantype dispersion. No diffusion between these streamlines and no diffusion/dispersion in the flow direction in a streamline is invoked. Along each streamline, however, matrix diffusion has affected the solute residence time differently due to different solute-advection time in each of the fictitious streamlines, which has been uniquely defined by the Fickian dispersion coefficient; hence, the solution to the single-flow-path model suggests that Fickian-type dispersion and velocity dispersion can be treated by the same formalism, and can therefore be combined to include both the mechanisms.

For this reason, the new solution is extended into more general cases where a discrete or continuous set of flow channels is present in fractures in rocks, similar to the channel network model (Gylling et al. 1999). This leads to a development of the multi-channel model in contrast to the singleflow-path model. The difference between these two models lies essentially in how these approaches deal with hydrodynamic dispersion. The single-flow-path model accounts only for Fickian-type dispersion due to diffusion across the aperture of the channels with constant aperture. The multi-channel model considers, however, both Fickian-type dispersion for transverse mixing across the cross section of, e.g. tapered channels, and velocity dispersion, resulting from different flow rates of the channels. As a result, the general solution developed for the multi-channel model, as well as the approximated solution, would find more applications in the interpretation of field tracer tests.

As expected, the analysis suggests that the use of the single-flow-path model for practical applications is questionable because its predictions are in clear violation to field observations (Gelhar et al. 1992; Gelhar 1993; Hjerne et al. 2010). By contrast, the multi-channel model accounting for both Fickian and velocity dispersions behaves very differently and it gives predictions in good agreement with what was observed in field tracer tests. In addition, it is demonstrated that short-range experiments cannot be used to determine dispersion mechanisms over large distances, unless it is known that spreading of a tracer pulse is caused only by Fickian-type dispersion and that there is no contribution from velocity dispersion.

One novelty of this contribution is, therefore, the new simple and robust solution to the single-flow-path model that 
accounts for the effects of advection, Fickian-type dispersion and matrix diffusion on solute transport in fractured crystalline rocks. Another novelty of this study is the extension of this solution into more general cases where Fickian-type dispersion and velocity dispersion can both be taken into account, leading to a development of the multi-channel model. The third novelty is the introduction of new expressions describing the Taylor-like dispersion for transverse mixing in tapered and sinusoidal wave-shaped channels. The fourth novelty of this paper is the statistical description of the apertures and the flow rates of the ensemble of channels. Based on the results of this study, it would appear that the multi-channel model provides a new general and efficient tool to understand and predict dispersion effects on solute transport in fractured rocks.

The remainder of this contribution is organized as follows. In the next section, the model proposed by Tang et al. (1981) is briefly presented along with the mathematical formulation of the problem. The derivation of the new solution to the model is then detailed followed with physical interpretations of the solution and a discussion on numerical implementations. In section 'Non-Fickian dispersion due to velocity variation between channels', an extension of the solution into more general cases is made where it is shown how to include both the Fickian-type and velocity dispersions into the multi-channel model, accounting especially for transverse mixing within and along a flow path. Different behaviors of the classical and the multi-channel models in predicting the breakthrough curves of a tracer pulse are then illustrated, together with a sensitivity analysis for the performance of the multi-channel model. The contribution ends with concluding remarks.

\section{Mathematical model and solution procedure}

The system under consideration is relatively simple compared to those studied by, e.g. Shahkarami et al. (2015) and Mahmoudzadeh et al. (2013). It suffices, however, for the need in the analysis of field tracer tests, and should be taken as the starting point before going deeper into complicated systems involving, e.g. either different geological layers or stagnant water zones.

As schematically shown in Fig. 1, the system under study is the same system as that conceptualized by Tang et al. (1981) for solute transport in fractured crystalline rock. It consists of a single fracture represented by two smooth parallel plates with a constant aperture $2 b$, embedded in an infinite and homogeneously porous rock. The water velocity in the fracture is assumed constant, and a pulse of tracer is injected over a certain period of time at the origin of the fracture.

Based on the same assumptions and simplifications of Tang et al. (1981), the transport processes in the single fracturematrix system can be described by two coupled, onedimensional (1D) equations, along with the initial and

\section{$z$}
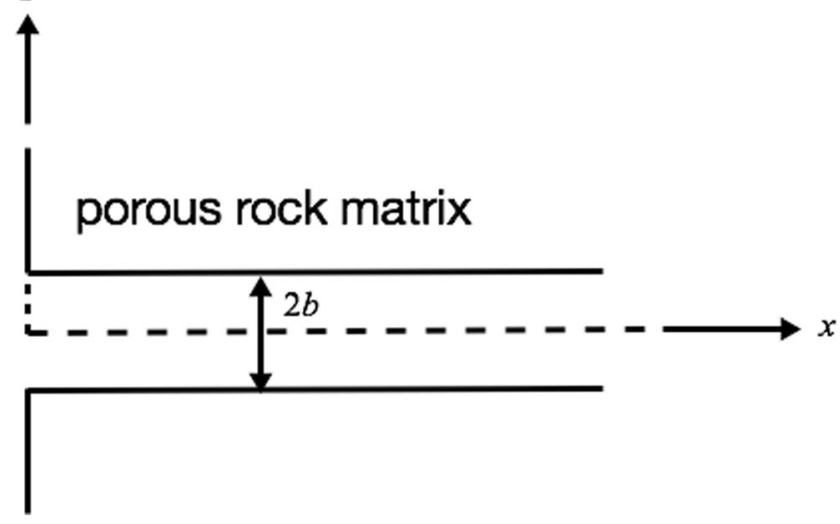

Fig. 1 Schematic view of the fracture-matrix system

boundary conditions. For the sake of completeness, the equations of continuity in the fracture and porous matrix are provided here. The governing equation in the fracture is as follows

$R_{\mathrm{f}} \frac{\partial c_{\mathrm{f}}}{\partial t}=-u \frac{\partial c_{\mathrm{f}}}{\partial x}+D_{\mathrm{f}} \frac{\partial^{2} c_{\mathrm{f}}}{\partial x^{2}}+\left.D_{\mathrm{p}} \varepsilon_{\mathrm{p}} \frac{1}{b} \frac{\partial c_{\mathrm{p}}}{\partial z}\right|_{z=b}-R_{\mathrm{f}} \lambda c_{\mathrm{f}}$

with the initial and boundary conditions given by:

$c_{\mathrm{f}}(x, 0)=0$

$c_{\mathrm{f}}(0, t)=c_{\text {in }}(t)$

$c_{\mathrm{f}}(\infty, t)=0$

and the diffusion equation in the porous matrix is

$R_{\mathrm{p}} \frac{\partial c_{\mathrm{p}}}{\partial t}=D_{\mathrm{p}} \frac{\partial^{2} c_{\mathrm{p}}}{\partial z^{2}}-R_{\mathrm{p}} \lambda c_{\mathrm{p}}$

with the initial and boundary conditions given by:

$c_{\mathrm{p}}(x, z, 0)=0$

$c_{\mathrm{p}}(x, b, t)=c_{\mathrm{f}}(x, t)$

$c_{\mathrm{p}}(x, \infty, t)=0$

where the subscripts $\mathrm{f}$ and $\mathrm{p}$ refer to the fracture and pore space of the rock matrix, respectively; $x$ and $z$ are the coordinates along and perpendicular to the fracture plane, respectively; $t$ denotes the time; $c$ represents the concentration of the tracer; $R$ the retardation coefficient; $D$ the coefficient of longitudinal dispersion in the fracture or the coefficient of pore diffusion in the homogeneous rock matrix; $u$ the groundwater velocity in the fracture; $\varepsilon$ the porosity; $\lambda$ the decay constant; and $c_{\mathrm{in}}(t)$ describes the concentration of the tracer at the origin of the fracture as a function of time.

By applying the Laplace transform to the system of governing equations, along with the initial and boundary 
conditions, it can be justified that the solution to $c_{\mathrm{f}}$ in the Laplace domain can be written as (Tang et al. 1981)

$\bar{c}_{\mathrm{f}}=\bar{c}_{\mathrm{in}} \exp \left(\frac{\mathrm{Pe}}{2}\right) \exp \left[-\frac{\mathrm{Pe}}{2} \sqrt{1+\frac{4}{\mathrm{Pe}} t_{\mathrm{w}}\left(G \sqrt{S}+R_{\mathrm{f}} S\right)}\right]$

This is called the basic solution where $\bar{c}_{\mathrm{f}}$ and $\bar{c}_{\text {in }}$ are the Laplace transformation of $c_{\mathrm{f}}$ and $c_{\mathrm{in}}$, respectively; $t_{\mathrm{w}}$ is the mean water residence time: $t_{\mathrm{w}}=x / u$; Pe is the Peclet number: $\mathrm{Pe}=u x / D_{\mathrm{f}}$, and $S=s+\lambda$ with $s$ being the Laplace variable. In writing this equation, the diffusive mass-transfer parameter $G$ is defined as

$G=\mathrm{MPG} / b$

with the material property group for the rock matrix given by (Mahmoudzadeh et al. 2013, 2016)

$$
\mathrm{MPG}=\varepsilon_{\mathrm{p}} \sqrt{R_{\mathrm{p}} D_{\mathrm{p}}}
$$

Since both $S$ and $\sqrt{S}$ appear inside the square root, it is normally difficult to evaluate its inverse transform. Nevertheless, it is possible to make use of the identity (Tang et al. 1981):

$\exp (-2 \chi)=\frac{2}{\sqrt{\pi}} \int_{0}^{\infty} \exp \left(-\xi^{2}-\frac{\chi^{2}}{\xi^{2}}\right) \mathrm{d} \xi$

to rewrite Eq. (9) as

$\bar{c}_{\mathrm{f}}=\exp \left(\frac{\mathrm{Pe}}{2}\right) \frac{2}{\sqrt{\pi}} \int_{0}^{\infty} \exp \left(-\xi^{2}-\frac{\mathrm{Pe}^{2}}{16 \xi^{2}}\right) \bar{c}_{\mathrm{in}} \exp \left[-\frac{\mathrm{Pe}}{4 \xi^{2}} t_{\mathrm{w}}\left(G \sqrt{S}+R_{\mathrm{f}} S\right)\right] \mathrm{d} \xi$

In what follows, Eq. (13) is referred to as the solution of Tang et al. (1981) in the Laplace domain. Taking this equation as a footstone of derivation, they ended up with an expression for $c_{\mathrm{f}}$ in the case of a Heaviside step injection. It looks similar to Eq. (13) in form but more complex in structure, involving a $\xi$-dependent variable that lumps the pore diffusion coefficient $D_{\mathrm{p}}$ with the longitudinal dispersion coefficient $D_{\mathrm{f}}$. This makes it difficult to understand the roles of matrix diffusion and Fickian dispersion in affecting solute transport and how they interact with each other, not to mention that the variable of integration $\xi$ does not have an obvious physical meaning; furthermore, computational difficulties often arise when one attempts to apply the solution for practical use. The reason for this is due mainly to the form of an integral with an infinite upper limit that the solution takes, significantly increasing the computational burden when evaluated numerically for each point in space and time. One can, as suggested by Tang et al. (1981), first scan the integrand prior to integration to determine its range, and then locate the integration points within the range to solve the problem, which may, however, make the numerical evaluation less efficient and apt to diverge in the results, especially in the cases involving low water velocities or large dispersion coefficients.

When convenient, the solution of Tang et al. (1981) can be used to distinguish the degree to which the measured breakthrough curve deviates from its predicted results, and consequently gives hints as to whether non-Fickian dispersion and diffusion took place; however, provided this is the case, it is incapable of changing the nature of its predictions because the inherent constraints as to the constant water velocity and constant dispersion coefficient cannot be relaxed. The solution of Tang et al. (1981) is, hence, not general in the sense that it cannot be extended into general cases where heterogeneity in either flow field or matrix diffusion has to be accounted for.

\section{General transient solution}

To overcome the aforementioned shortcomings and inadequacies, a different but simple strategy may be adopted to obtain the general transient solution for the problem of solute transport along a single fracture in a porous rock. In the following, it is shown that the new solution takes a simple form and has clear physical meaning, making it possible to account for both Fickian and velocity dispersions simultaneously.

\section{Solution to the single-flow-path model}

By introducing a new variable $\tau$ defined as:

$\tau=\frac{\mathrm{Pe}}{4 \xi^{2}} t_{\mathrm{w}}$

the solution of Tang et al. (1981), i.e. Eq. (13), becomes

$\bar{c}_{\mathrm{f}}=\int_{0}^{\infty} f(\tau) \bar{c}_{\text {in }} \exp \left[-\tau\left(G \sqrt{S}+R_{\mathrm{f}} S\right)\right] \mathrm{d} \tau$

with the $f$ function given by

$f(t)=\frac{1}{2 \sqrt{\pi}} \frac{1}{t} \sqrt{\frac{\mathrm{Pe} t_{\mathrm{w}}}{t}} \exp \left(\frac{\mathrm{Pe}}{2}-\frac{\mathrm{Pe}}{4} \frac{t_{\mathrm{w}}}{t}-\frac{\mathrm{Pe}}{4} \frac{t}{t_{\mathrm{w}}}\right)$

One may note that $f(t)$ function does not contain any items that depend on $s$ or $S$. Later, it will be shown that the $f(t)$ is, in fact, the system response to a pulse injection when no matrix diffusion, no chemical sorption and no decay are invoked. For now, in order to further simplify the solution of Tang et al. (1981), it is of interest to consider the limiting case when 
$\mathrm{Pe} \rightarrow \infty$. In this case, it can readily be seen that the basic solution in the Laplace domain, i.e. Eq. (9) reduces to

$\left.\bar{c}_{\mathrm{f}}\left(t_{\mathrm{W}}\right)\right|_{\mathrm{Pe} \rightarrow \infty}=\bar{c}_{\mathrm{in}} \exp \left[-t_{\mathrm{W}}\left(G \sqrt{S}+R_{\mathrm{f}} S\right)\right]$

where $\bar{c}_{\mathrm{f}}\left(t_{\mathrm{w}}\right)$ has been used to emphasize the fact that $\bar{c}_{\mathrm{f}}$ is actually $t_{\mathrm{w}}$-dependent. For several practically important $c_{\mathrm{in}}(t)$ cases, the inverse transform for $\left.\bar{c}_{\mathrm{f}}\left(t_{\mathrm{w}}\right)\right|_{\mathrm{Pe} \rightarrow \infty}$ is straightforward and can generally be written as (see Appendix A)

$\left.c_{\mathrm{f}}\left(t, t_{\mathrm{w}}\right)\right|_{\mathrm{Pe} \rightarrow \infty}=g\left(t-R_{\mathrm{f}} t_{\mathrm{w}}, G t_{\mathrm{w}}\right) H\left(t-R_{\mathrm{f}} t_{\mathrm{w}}\right)$

where the arguments of the $g$ function implies that it depends partly on $\left(t-R_{\mathrm{f}} t_{\mathrm{w}}\right)$ and partly on $G t_{\mathrm{w}}$ only, and $H(t)$ is the Heaviside step function. Then, if one compare the expression for $\left.\bar{c}_{\mathrm{f}}\left(t_{\mathrm{W}}\right)\right|_{\mathrm{Pe} \rightarrow \infty}$ in Eq. (17) with the integrand in Eq. (15) and place the expression for $\left.\bar{c}_{\mathrm{f}}\left(t_{\mathrm{w}}\right)\right|_{\mathrm{Pe} \rightarrow \infty}$ in Eq. (15), the latter can be rewritten as

$\bar{c}_{\mathrm{f}}=\left.\int_{0}^{\infty} f(\tau) \bar{c}_{\mathrm{f}}(\tau)\right|_{\mathrm{Pe} \rightarrow \infty} \mathrm{d} \tau$

It is noted that the integrand of the above expression is a product of the function $f(\tau)$, which is independent of the Laplace variable, $s$, and a function $\left.\bar{c}_{\mathrm{f}}(\tau)\right|_{\mathrm{Pe} \rightarrow \infty}$, which describes the influence of matrix diffusion and surface retardation in the Laplace domain. As a result, the inverse transform for $\bar{c}_{\mathrm{f}}$ gives

$c_{\mathrm{f}}(t)=\left.\int_{0}^{\infty} f(\tau) c_{\mathrm{f}}(t, \tau)\right|_{\mathrm{Pe} \rightarrow \infty} \mathrm{d} \tau$

or equivalently, using Eq. (18),

$c_{\mathrm{f}}(t)=\int_{0}^{t / R_{\mathrm{f}}} f(\tau) g\left(t-R_{\mathrm{f}} \tau, G \tau\right) \mathrm{d} \tau$

In the remainder of this study, Eq. (21) is referred to as the solution to the single-flow-path model. As detailed in Appendix A, the analytical solutions to $\left.c_{\mathrm{f}}(t, \tau)\right|_{\mathrm{Pe} \rightarrow \infty}$ are always analytically available provided that the rock matrix is homogeneous and the input source is defined by the evolution of solute concentration over time. In particular, in the case of a Dirac delta injection, the function $g$ becomes (see Appendix A)

$g\left(t-R_{\mathrm{f}} t_{\mathrm{w}}, G t_{\mathrm{w}}\right)=m_{0} \frac{G t_{\mathrm{w}}}{2 \sqrt{\pi\left(t-R_{\mathrm{f}} t_{\mathrm{w}}\right)^{3}}} \exp \left[-\frac{\left(G t_{\mathrm{w}}\right)^{2}}{4\left(t-R_{\mathrm{f}} t_{\mathrm{w}}\right)}-\lambda t\right]$

where $G t_{\mathrm{w}}$ is actually $\mathrm{MPG} \cdot \mathrm{FWS} / Q$, provided FWS is the area of flow-wetted-surface of the single fracture (Mahmoudzadeh et al. 2013) over which solute is exchanged with the stagnant water in the rock matrix, and $Q$ is the volumetric flow rate through the fracture.

\section{Decoupled effect of matrix diffusion and Fickian dispersion}

In what follows, some useful insights are provided into the solution derived for the single-flow-path model. Consider the simple case when $m_{0}=1$ and $\lambda=0$. The function $g(t)$ then becomes

$g\left(t-R_{\mathrm{f}} t_{\mathrm{w}}, G t_{\mathrm{w}}\right)=\frac{G t_{\mathrm{w}}}{2 \sqrt{\pi\left(t-R_{\mathrm{f}} t_{\mathrm{w}}\right)^{3}}} \exp \left[-\frac{\left(G t_{\mathrm{w}}\right)^{2}}{4\left(t-R_{\mathrm{f}} t_{\mathrm{w}}\right)}\right]$

therefore, for a fracture-only system without matrix diffusion, i.e. $G=0$, the function $g$ reduces to

$g\left(t-R_{\mathrm{f}} t_{\mathrm{w}}, G t_{\mathrm{w}}\right)=\delta\left(t-R_{\mathrm{f}} t_{\mathrm{w}}\right)$

This result can directly be obtained from the expression for $\left.\bar{c}_{\mathrm{f}}\left(t_{\mathrm{W}}\right)\right|_{\mathrm{Pe} \rightarrow \infty}$, i.e. Eq. (17). In this special case with $c_{\mathrm{in}}=\delta(t)$, $\lambda=0$ and $G=0$, Eq. (17) gives: $\left.\bar{c}_{\mathrm{f}}\left(t_{\mathrm{W}}\right)\right|_{\mathrm{Pe} \rightarrow \infty}=\exp \left(-R_{\mathrm{f}} t_{\mathrm{W}} s\right)$. Then, the inverse transform for $\left.\bar{c}_{\mathrm{f}}\left(t_{\mathrm{W}}\right)\right|_{\mathrm{Pe} \rightarrow \infty}$ yields, immediately, the expression in Eq. (24). Furthermore, it follows that by replacing $t_{\mathrm{w}}$ with $\tau$ in Eq. (24) and then inserting into the solution to the single-flow-path model, Eq. (21), one obtains for a fracture-only system, i.e. a system with no matrix diffusion effect,

$c_{\mathrm{f}}(t)=f\left(t / R_{\mathrm{f}}\right)$

which indicates that $f(t)$ is nothing but the response to the Dirac delta injection in the special case involving no decay $(\lambda=0)$, no surface sorption $\left(R_{\mathrm{f}}=1\right)$ and no matrix diffusion $(G=0)$. In practice, it can also be justified from Eq. (16) that, by substituting the definitions of both Pe and $t_{\mathrm{w}}$ into the equation, $f(t)$ is indeed the solution to the advection-dispersion equation of a fracture-only system, i.e.

$f(t)=\frac{1}{2 \sqrt{\pi D_{\mathrm{f}} t}} \frac{x}{t} \exp \left[-\frac{(x-u t)^{2}}{4 D_{\mathrm{f}} t}\right]$

As a result, the variable $\tau$ of integration in the solution to the single-flow-path model, i.e. Eq. (21), can be understood as a solute residence time in the fracture-only system assuming $R_{\mathrm{f}}=1$ and $\lambda=0$. Since in deriving the transport equation in the fracture, Eq. (1), the water velocity $u$ is assumed constant, the $f(t)$ expression in Eq. (26) describes how the spread of solute residence time behaves at a given distance. Therefore, the variable $\tau$ in the solution to the single-flow-path model can also be thought of as a fictitious residence time of water that carries the solute.

Moreover, one may note that in the case of a plug flow without any dispersion/diffusion, i.e. $D_{\mathrm{f}}=0$, the Gaussian distribution function $f(t)$ becomes the Dirac delta function $f(t)=\delta\left(t-t_{\mathrm{w}}\right)$; therefore, $c_{\mathrm{f}}(t)$ in Eq. (21) reduces to 
$\left.\bar{c}_{\mathrm{f}}\left(t_{\mathrm{W}}\right)\right|_{\mathrm{Pe} \rightarrow \infty}$, which verifies that the $g(t)$ function is really the solution to $c_{\mathrm{f}}(t)$ in the limiting case as $\mathrm{Pe} \rightarrow \infty$..

In more general cases, the solution to the single-flow-path model, Eq. (21), indicates that $c_{\mathrm{f}}(t)$ is seemingly the convolution of $f(t)$ and $g(t)$. The consequence is equivalent to splitting the single fracture-matrix system shown in Fig. 1 into two subsystems with different transport mechanisms. As shown in Fig. 2, the $f(t)$ function is the response of the fracture-only system accounting merely for advection and dispersion to the $\delta(t)$ injection, i.e.

$f(t)=\left.c_{\mathrm{f}}\right|_{\delta, R_{\mathrm{f}}=1, \lambda=0, D_{\mathrm{p}}=0}$

where the subscript $\delta$ refers to the case of a Dirac delta injection with $m_{0}$ taken to be unity.

The $g(t)$ function is the response of the fracture-matrix system involving advection, matrix diffusion, sorption and decay but no dispersion to the $c_{\text {in }}(t)$ injection, given a solute-advection time $\tau$ (the distribution of which is defined by the $f$ function), i.e.

$g(t)=\left.c_{\mathrm{f}}\right|_{c_{i n}, \tau, \mathrm{Pe} \rightarrow \infty}$

where the subscript $c_{\text {in }}$ refers to the actual injection case, and the subscript $\tau$ means that the system now has an solute-advection time $\tau$ instead of $t_{\mathrm{w}}$. Remember that $g(t)$ is an abbreviation of $g(t$ $\left.-R_{\mathrm{f}} \tau, G \tau\right)$ in Eq. (21).

One can, then, imagine that there are numerous nondispersion systems with plug flows where hydrodynamic dispersion has been completely neglected. Each of these systems has, however, a different solute-advection time, which follows a distribution defined by the $f(t)$ function. The seemingly convolution of $f(t)$ and $g(t)$ according to Eq. (21) gives, then, the solution for $c_{\mathrm{f}}(t)$ at the outlet of the fracture, i.e. the solution to the single-flow-path model. As a result, the effects of hydrodynamic dispersion and matrix diffusion on solute transport have been decoupled in the sense that these two transport mechanisms can be viewed separately and independently. The $f(t)$ function describes practically the probability density function of the residence time distribution of a conservative solute resulting merely from advection and Fickian dispersion. The $g(t)$ function characterizes the mass exchange between the flow fracture and the porous rock matrix, given a soluteadvection time. It should be note that this formulation is consistent with the stochastic-convective transport theory (Ginn 2001) that was developed to treat dispersion and non-linear reaction in heterogeneous media (Simmons et al. 1995).

\section{Analogy with the velocity dispersion model formulation}

Noticeably, the $f(t)$ function for solute residence time distribution of a fracture-only system can also be imagined as a result of the solutes having fictitiously followed streamlines with different constant velocities. The solute-advection time along the streamlines is uniquely defined by Fickian dispersion coefficient $D_{\mathrm{f}}$. No diffusion between these fictitious streamlines and no diffusion/dispersion in the flow direction in a streamline is now invoked since $D_{\mathrm{f}}$ already includes the effect of diffusion between streamlines caused by, e.g. Taylor dispersion. Such dispersion is and must be Fickian for Eq. (1) to be valid. Then, as sketched in Fig. 3, one may consider the fracture-matrix system as one composed of a series of stream tubes with different flow rates $q_{\mathrm{f}}$, the distribution of which can be represented by a continuous function $h(q)$. The water has, then, different residence time in each of the stream tubes, which is inversely proportional to $q$, but encounters the same area of flow-wetted-surface. The dispersion of the solute along each stream tube is caused, however, only by matrix diffusion.

It follows that it is possible to relate the flow rate $q_{\mathrm{f}}$ of each of the fictitious stream tubes to the solute-advection time $\tau$, the variable of integration in Eq. (21), by

$q_{\mathrm{f}}=q_{\mathrm{w}} \frac{t_{\mathrm{w}}}{\tau}$

where $q_{\mathrm{w}}$ denotes the mean volumetric flow rate of the stream tubes.
Fig. 2 Decoupling the fracture-

matrix system into two subsystems with different transport mechanisms (a)

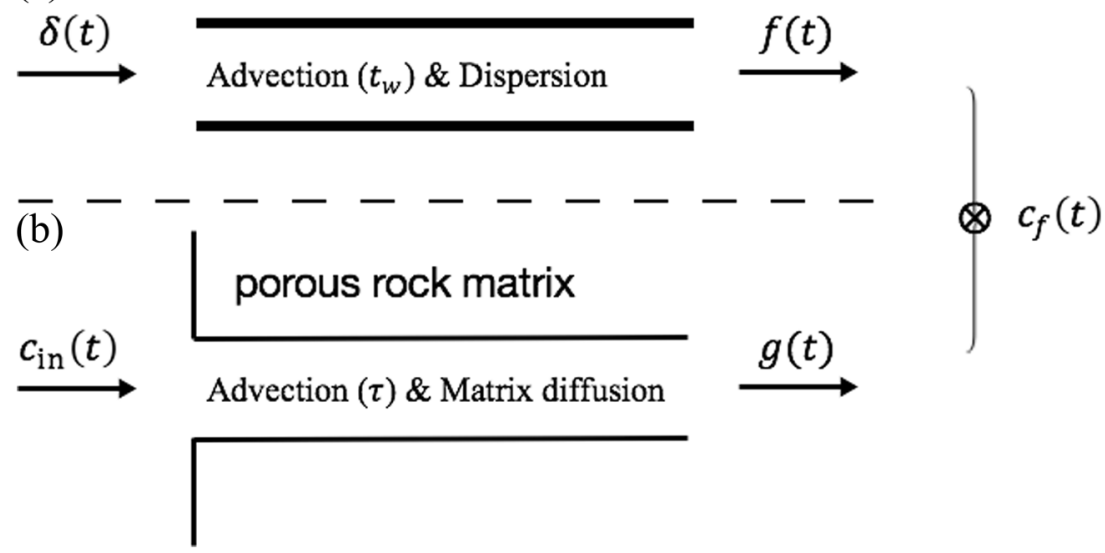




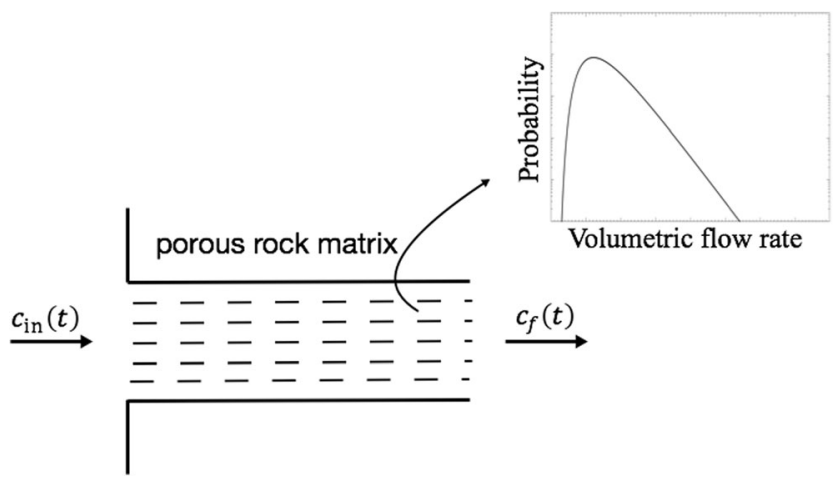

Fig. 3 Interpretation of the process of solute transport in the fracturematrix system by the independent stream-tube approach

This allows the solution to the single-flow-path model, Eq. (21), to be rewritten as

$c_{\mathrm{f}}(t)=\int_{R_{\mathrm{f}} t_{\mathrm{w}} q_{\mathrm{w}} / t}^{\infty} h\left(q_{\mathrm{f}}\right) g\left(t-R_{\mathrm{f}} t_{\mathrm{w}} q_{\mathrm{w}} / q_{\mathrm{f}}, G t_{\mathrm{w}} q_{\mathrm{w}} / q_{\mathrm{f}}\right) \mathrm{d} q_{\mathrm{f}}$

with the $h(q)$ function given by

$h(q)=\frac{t_{\mathrm{w}} q_{\mathrm{w}}}{q^{2}} f\left(\frac{t_{\mathrm{w}} q_{\mathrm{w}}}{q}\right)$

Upon the use of $f(t)$ expression in Eq. (26), it can be calculated that

$h(q)=\frac{1}{2 \sqrt{\pi}} \sqrt{\frac{\mathrm{Pe}}{q q_{\mathrm{w}}}} \exp \left[-\frac{\operatorname{Pe}}{4} \frac{\left(q-q_{\mathrm{w}}\right)^{2}}{q q_{\mathrm{w}}}\right]$

whereby the $h(q)$ function describes the flow rate distribution of the fictitious stream tubes caused by Fickian dispersion.

Interestingly, as a variant form of Eq. (21), Eq. (30) is formulated in the same way as that underlying the concept of the velocity dispersion model (Neretnieks 1983; Chesnut 1994), namely by collecting and mixing the solute from different real stream tubes with different flow rates and water residence times, which have been subject to different spreading mechanisms along each of the stream tubes. These real stream tubes can, e.g. be the different flow paths, channels, in a fractured rock leading from A to B where no mixing or exchange of solute takes place between the flow paths. This spreading behavior is, however, quite different from Fickian dispersion.

\section{Accuracy and robustness of the solution}

Before proceeding, it is worth noting that compared to the solution of Tang et al. (1981), both limits of integration in the solution of the single-flow-path model, Eq. (21), are now finite. Due to the nice property of the $f(t)$ function, the upper limit can further be reduced significantly, taking as $\min \left(t / R_{\mathrm{f}}, t_{\mathrm{w}}+3 \sigma_{\mathrm{t}}\right)$ with $\sigma_{\mathrm{t}}=$ $\sqrt{2 t_{\mathrm{w}} / \mathrm{Pe}}\left(\sigma_{\mathrm{t}}\right.$ can be thought of being an estimate of the deviation of the $f(t)$ function with respect to time). This makes numerical integration of the new solution much more stable and efficient, without the need to scan the integrand prior to integration as that required by the solution of Tang et al. (1981).

To demonstrate the accuracy of the new solution, the results of Eq. (21) are compared to the breakthrough curves obtained from the solution of Tang et al. (1981) as well as to the results from a numerical approach that transforms the basic solution, Eq. (9), back to the time domain by the De Hoog algorithm (De Hoog et al. 1982). The simulations are made in the case of a Heaviside step injection, for which the expression for $\left.c_{\mathrm{f}}(t, \tau)\right|_{\mathrm{Pe} \rightarrow \infty}$ is presented in Appendix A, Eq. (51). The simulation results are presented in Fig. 4 for a fracture at $x=$ $0.76 \mathrm{~m}$. The other parameters used are taken from the studies of Tang et al. (1981): $2 b=120 \mu \mathrm{m}, \varepsilon_{\mathrm{p}}=0.35, u=0.75 \mathrm{~m} / \mathrm{d}$, $D_{\mathrm{f}}=6.6 \times 10^{-6} \mathrm{~m}^{2} / \mathrm{s}, D_{\mathrm{p}}$ is varied in the range from 0.0 to $10^{-10} \mathrm{~m}^{2} / \mathrm{s}$, and radioactive decay and surface retardations are not considered.

Clearly, excellent agreement between the two analytical solutions and the numerical solution of the inverse Laplace
Fig. 4 Breakthrough curves for a fracture at $x=0.76 \mathrm{~m}$, for $D_{\mathrm{p}}=0.0,10^{-14}, 10^{-13}, 10^{-12}$, $10^{-11}$ and $10^{-10} \mathrm{~m}^{2} / \mathrm{s}$ (from top to bottom), respectively; the concentration is normalized by $c_{0}$

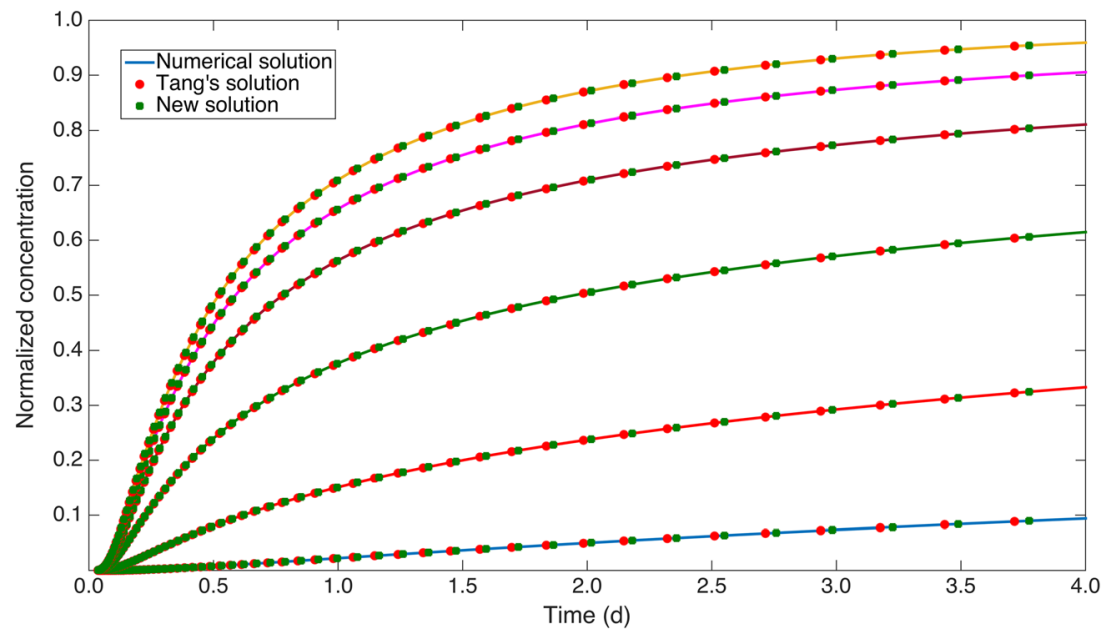


transform are obtained. In using the new form of the solution, however, only 20 Gauss points are required to give an integration that is virtually exact, in contrast to 50 Gauss points commonly used for the solution of Tang et al. (1981), when integration is performed by Gaussian quadrature. By comparison, the variant form of Eq. (21), i.e. Eq. (30), is not competitive because it is similar to the solution of Tang et al. (1981) also having an infinite upper limit. For this reason, the authors will only discuss the use of Eq. (21) in the following.

In addition, it is worth emphasizing that the solution to the single-flow-path model has been developed under the conceptualization that the fracture has a constant aperture $2 b$ and is fully conductive for water flow with a constant velocity $u$. This idealization allows for the evaluation of the dispersion coefficient $D_{\mathrm{f}}$ by Taylor dispersion theory (Taylor 1953; Bird et al. 2002) that accounts for the effect of diffusion across the aperture of the fracture, to give

$D_{\mathrm{f}}=D_{\mathrm{w}}+\frac{u^{2} b^{2}}{52.5 D_{\mathrm{w}}}$

where $D_{\mathrm{w}}$ is the molecular diffusion coefficient of the solute in water.

The dispersion due to diffusion across the aperture of the flow channel (small part of the fracture, as discussed in the next section) is, however, often very small and can be neglected compared to that across the width of the variableaperture channels. The diffusion between streamlines at different locations over the width of the channel in the fracture perpendicular to the flow direction would be more important than that across the aperture because the flow channels are much wider than the apertures. To account for this effect, Taylor dispersion theory (Taylor 1953; Bird et al. 2002) can also be applied. As detailed in Appendix B, for a channel with rhomboidal or wave-shaped cross section, it can be written

$D_{\mathrm{f}}=D_{\mathrm{w}}+\frac{u^{2} W^{2}}{C_{\mathrm{D}} D_{\mathrm{w}}}$

where $W$ is the half width of the channel, and the constant $C_{\mathrm{D}}=$ 48 and 77.9 for the rhomboidal and wave-shaped channels, respectively. When compared to Eq. (33), this result suggests that transverse mixing across the width of the channels may take much longer time to even out the concentration difference between the streamlines, but when it does, quite large Fickian dispersion could be caused - unfortunately, however, there is very little data on the widths and width distributions of channels.

Moreover, it should be noted that in all the cases studied in this paper, it is assumed that the Fickian dispersion has been fully developed in the channels. When this is not the case, the ADE-based models, e.g. Eq. (1), would not be valid as they lack the term describing transverse dispersion perpendicular to the flow direction. The authors will, nevertheless, not discuss this issue further as it is out of the scope of the present paper; instead, the focus is directed toward how to extend the new solution for practical use.

\section{Non-Fickian dispersion due to velocity variation between channels}

\section{The multi-channel model}

In contrast to the idealized picture sketched in Fig. 1, field observations indicate that fracture surfaces are uneven (Keller 1997) and water flows only in a small part of a conductive fracture to form one or more flow channels with variable aperture, as schematically shown in Fig. 5 (Tsang and Neretnieks 1998). To account for the effect of channeling on solute transport, the fracture might be seen as a system of $N$ independent channels assuming that the solute does not mix between the channels (Neretnieks 2002). A discrete form of the general transient solution for the solute concentration at the outlet of the fracture can, therefore, be written as

$c_{\mathrm{f}}(t)=\frac{\sum_{i=1}^{N} q_{i} c_{\mathrm{f}, i}(t)}{\sum_{i=1}^{N} q_{i}}$

where the summation runs over the number of the flow channels that make up of the fracture, and $c_{\mathrm{f}, i}(t)$ denotes the concentration at the outlet of the $i$ th channel.

The assumption behind this equation is that any influence on $c_{\mathrm{f}, i}$ by $q_{i}$ is already accounted for, and that there may be different transport and interaction mechanisms along different channels. However, assuming Eq. (1) holds for each of the channels where Fickian dispersion is active, it is possible to describe $c_{\mathrm{f}, i}(t)$ by an

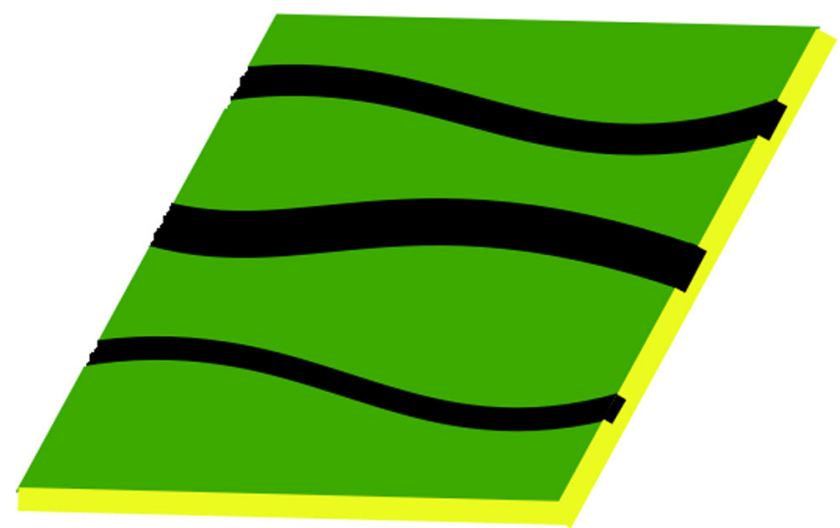

Fig. 5 Schematic view of the flow channels in a conductive fracture 
expression analogous to the solution to the single-flowpath model, i.e.

$c_{\mathrm{f}, i}(t)=\int_{0}^{t / R_{\mathrm{f}}} f_{i}(\tau) g_{i}\left(t-R_{\mathrm{f}} \tau, G \tau\right) \mathrm{d} \tau$

When substituting this expression into Eq. (35), it can be written

$c_{\mathrm{f}}(t)=\frac{1}{\sum_{i=1}^{N} q_{i}} \int_{0}^{t / R_{\mathrm{f}}} \sum_{i=1}^{N} q_{i} f_{i}(\tau) g_{i}\left(t-R_{\mathrm{f}} \tau, G \tau\right) \mathrm{d} \tau$

Clearly, $c_{\mathrm{f}}(t)$ cannot be expressed in the form of an apparent convolution anymore in the multi-channel cases; nevertheless, the discrete form of the solution illustrates the basic principles to account for the effect of channeling or velocity dispersion on solute transport in fractured rocks. In practice, however, it is almost impossible to know the number and properties of channels that may present in natural fractures. For this reason, and also for facilitating mathematical manipulations, a continuous form of Eq. (35) is more preferable by assuming that there are numerous channels with significant changes in the flow rates, instead of a few distinct channels, along the flow path. This leads to a similar physical conceptualization of solute transport in a fracture as that illustrated in Fig. 3. However, the flow components of the open fracture should now be understood as (macro) flow channels, instead of stream tubes (to avoid confusion, it is here presumed that macro channels with average properties are made up of micro stream tubes; one can of course take the channels also as macro stream tubes). The effect is a natural extension of Eq. (35), i.e. the solution to $c_{\mathrm{f}}(t)$ now becomes (Neretnieks 2002),

$c_{\mathrm{f}}(t)=\frac{1}{q_{\text {mean }}} \int_{0}^{\infty} c_{\mathrm{f}}(t ; q) \kappa(q) q \mathrm{~d} q$

where $c_{\mathrm{f}}(t ; q)$ denotes the solute concentration in the channel having a volumetric flow rate $q, \kappa(q)$ describes the probability distribution of the volumetric flow rate of the channels, and $q_{\text {mean }}$ is the mean flow rate of the ensemble of channels defined as

$q_{\text {mean }}=\int_{0}^{\infty} \kappa(q) q \mathrm{~d} q$

Thus, contrary to $h\left(q_{\mathrm{f}}\right)$ in the single-flow-path model that represents the flow rate distribution of the fictitious (micro) stream tubes passing through each of the channels, $\kappa(q)$ represents the real flow rate distribution among the channels. $\kappa(q)$ for the flow channels with different flow rates should, therefore, be estimated from the aperture field of natural fractures, which mostly follows a log-normal distribution (Hakami and Larsson 1996; Keller 1997). This is exemplified in studies by
Liu and Neretnieks $(2005,2006)$ and Moreno et al. (1988) where the spatial variations of the aperture field is taken into account to assess the stochastic properties of fluid flow in real fractures.

In an idealized case when solute concentration along all the channels, i.e. $c_{\mathrm{f}}(t ; q)$, can be described by the solution to the single-flow-path model, given in Eq. (21), Eq. (38) becomes

$c_{\mathrm{f}}(t)=\frac{1}{q_{\text {mean }}} \int_{0}^{\infty} \int_{0}^{t / R_{\mathrm{f}}} f(\tau ; q) g\left(t-R_{\mathrm{f}} \tau, G \tau ; q\right) \kappa(q) q \mathrm{~d} \tau \mathrm{d} q$

where $q$ is also introduced at the right-hand side of the semicolon to stress that the $f(t)$ and $g(t)$ functions implicitly depend on the volumetric flow rate $q$ via either $u$ or $b$. To make it clearer, throughout the paper, Eq. (40) is referred to as the solution to the multi-channel model.

This result is actually the solution to $c_{\mathrm{f}}(t)$ obtained by multiplying the transport equation in a single flow path (or channel), i.e. Eq. (1), with $q$ and then integrating over all the channels with different flow rates. In other words, it gives the joint residence time distribution of a tracer pulse or the breakthrough curves at the point of observation by solving the following equation:

$R_{\mathrm{f}} \frac{\partial c_{\mathrm{f}}}{\partial t}=-\frac{1}{q_{\text {mean }}} \int_{0}^{\infty}\left(u \frac{\partial c_{\mathrm{f}}}{\partial x}-D_{\mathrm{f}} \frac{\partial^{2} c_{\mathrm{f}}}{\partial x^{2}}-\left.D_{\mathrm{p}} \varepsilon_{\mathrm{p}} \frac{1}{b} \frac{\partial c_{\mathrm{p}}}{\partial z}\right|_{z=b}\right) \kappa(q) q \mathrm{~d} q-R_{\mathrm{f}} \lambda c_{\mathrm{f}}$

which is coupled with the transport equation in the porous matrix, Eq. (2), along with the given initial and boundary conditions. Note, however, that the solute residence time distribution in each of the flow channels is still governed by the solution to the single-flow-path model, i.e. Eq. (21), which accounts for advection, Fickian dispersion and matrix diffusion. The solute does not mix between the channels, as mentioned earlier, and the Fickian dispersion coefficient in each of the channels should now be described by Eq. (34) to account for transverse mixing across the width of, e.g. tapered channels.

This independent multi-channel approach (Neretnieks 2002) is, as discussed in the preceding, in line with the concept of velocity dispersion and is based on the important argument that field scale experiments in fractured rocks are rarely (if ever) governed by a Fickian dispersion, notwithstanding the fact that the breakthrough curves for a given experiment can often be fitted reasonably well by the advection-dispersion equation. However, compiled data from a multitude of field experiments (Gelhar et al. 1992; Gelhar 1993) showed that the evaluated dispersion coefficient increases proportionally to the distance of observation, which is in clear contradiction to the assumption that $D_{\mathrm{f}}$ must be a constant for the ADE to be valid. 
The difference between Fickian and velocity dispersion can be illustrated by how a tracer pulse spreads with increasing distance. When Fickian dispersion dominates, the variance (i.e. the second moment) of the breakthrough curves increases in proportion to the distance traveled. When velocity dispersion dominates, however, it increases in proportion to the distance squared, i.e. much faster (Neretnieks 1983). At short distances, Fickian dispersion dominates and is increasingly overshadowed by velocity dispersion, which can, therefore, cause important differences when predicting solute transport over long distances based on the data obtained from field experiments over short distances.

\section{Statistical description of the flow rates}

Following this discussion, the authors go one step further to relate the $q$ distribution of the channels to the aperture field of fractures by the generalized the cubic law of laminar flow in a slit (Bird et al. 2002; Neretnieks 2002). As detailed in Appendix $\mathrm{C}$, under the conditions that the mean aperture $\bar{a}$ of the channels could well be described by a log-normal distribution function and that the natural fracture is composed of channels with identical geometries, the relation between $b$ and $\bar{a}, W$ and $\bar{a}, q$ and $\bar{a}, u$ and $\bar{a}$, are all available, as given in Eqs. (70), (85), (86) and (87), respectively. This allows the solution to the multi-channel model, Eq. (40), to be rewritten as

$c_{\mathrm{f}}(t)=\frac{1}{q_{\text {mean }}} \int_{0}^{\infty} \int_{0}^{t / R_{\mathrm{f}}} f\left(\tau ; u, D_{\mathrm{f}}\right) g\left(t-R_{\mathrm{f}} \tau, G \tau ; b\right) q(\bar{a}) p(\bar{a}) \mathrm{d} \tau \mathrm{d} \bar{a}$

This is called the exact analytical solution where $p(\bar{a})$ is the probability distribution function of the mean aperture of the flow channels, as given in Eq. (68). The difference between the mean aperture $\bar{a}$ and the aperture $2 b$ has been discussed in Appendix C. In brief, $\bar{a}$ is the mean of the real local apertures of each of the channels, whereas $2 b$ is an aperture that is not real but is a measure of the flow-wetted-surface area per unit flow-volume of the channel. Both would be identical for rectangular channels but different for tapered channels.

In writing Eq. (42), it is emphasized that the $f(t)$ function depends explicitly on $u$ and $D_{\mathrm{f}}$, and the $g(t)$ function depends on the half-aperture $b$ due to the $G$ parameter. Because of the dependence of $u, D_{\mathrm{f}}$ and $b$ on $\bar{a}$, no simple solution can be found for $c_{\mathrm{f}}(t)$ from Eq. (42); however, for non-sorbing solutes, a good approximation can be made in the high flow-rate cases by introducing:

$g\left(t-R_{\mathrm{f}} \tau, G \tau ; b\right) \approx g\left(t-R_{\mathrm{f}} \tau, G \tau ; b_{\text {mean }}\right)$

with the mean half-aperture $b_{\text {mean }}$ related to the mean aperture $a_{\text {mean }}$ by, as detailed in Appendix C,

$b_{\text {mean }}=C_{\mathrm{s}}\left(\frac{a_{\text {mean }}}{2}\right)$ where $C_{\mathrm{s}}$ is a shape factor accounting for the influence of the channel geometry on the flow conditions, and in practice $C_{\mathrm{s}} \approx$ 1 irrespective of the shape of the cross section of the channels.

As a result, the exact analytical solution, Eq. (42), can be rewritten as

$c_{\mathrm{f}}(t)=\int_{0}^{t / R_{\mathrm{f}}} f(\tau) g\left(t-R_{\mathrm{f}} \tau, G \tau ; b_{\text {mean }}\right) \mathrm{d} \tau$

with the joint $f(t)$ function now given by

$f(t)=\frac{1}{q_{\text {mean }}} \int_{0}^{\infty} f\left(\tau ; u, D_{\mathrm{f}}\right) q(\bar{a}) p(\bar{a}) \mathrm{d} \bar{a}$

Obviously, this approximated solution takes the same form as the solution to the single-flow-path model, given in Eq. (21). In fact, the joint $f(t)$ function and the averaged $g(t)$ function would both reduce, respectively, to the simple $f(t)$ and $g(t)$ functions obtained from the single-flow-path model, provided the fracture can be conceptualized as a single flow path. Due to its numerical efficiency, this approximated solution to the multi-channel model can be used to give a quick and qualitative analysis of field tracer tests. One should not, however, directly apply it to predict the transport of strongly sorbing solutes in fractured rocks.

The joint $f(t)$ function given in the previous also suggests that the effects of Fickian and velocity dispersions on the spreading of solute mass in natural fractures would be very different. The use of the single-flow-path model in the interpretation of field tracer tests would lead to a fundamental mistake. To illustrate this, the different behaviors of the single-flow-path and multi-channel models are discussed in the next section, especially for the extrapolation to other distances.

All nomenclature is listed in Appendix D.

\section{Simulations and discussion}

The system under study is the 1D transport of a tracer in a 2$\mathrm{m}$-long fracture surrounded by a porous rock matrix. The fracture is conceptualized to be composed of tapered channels with the mean half-width $W_{\text {mean }}=0.1 \mathrm{~m}$, and the mean aperture $a_{\text {mean }}=100 \mu \mathrm{m}$. The standard deviation of the aperture is chosen to be $\sigma=0.2135$ (corresponding to $\mathrm{Pe}=10$, as discussed in Appendix C), and the mean volumetric flow rate is set to $q_{\text {mean }}=0.2 \mathrm{ml} / \mathrm{d}$ (corresponding to $u=0.01 \mathrm{~m} / \mathrm{d}$ for a rectangular channel). The other parameters used in the simulations are taken from the studies of Tang et al. (1981): $D_{\mathrm{w}}=$ $1.6 \times 10^{-9} \mathrm{~m}^{2} / \mathrm{s}, D_{\mathrm{p}}=1.6 \times 10^{-10} \mathrm{~m}^{2} / \mathrm{s}, \varepsilon_{\mathrm{p}}=0.01, R_{\mathrm{p}}=1$, and $R_{\mathrm{f}}=1$. Unless otherwise indicated, these values apply to all the calculations presented in this section. It is also assumed that the channels are identical in width (i.e. $m=0$ ) and that the 
cubic law applies (i.e. $n=2$ ). In all cases, the results are presented as the solute concentration, normalized by $m_{0}$ (the ratio between the mass injected and the volumetric flow rate through the fracture), as a function of time for a stable species. The inclusion of radioactive decay in the calculations is trivial, but for the sake of highlighting the effects of both dispersion and matrix diffusion it is assumed that $\lambda=0$ throughout the studies. To facilitate the analysis, it is also convenient to only consider the case of a Dirac delta injection into the fracture.

\section{Accuracy of the approximate solution}

To start with, the accuracy of the approximated solution given in Eq. (45) in calculating the breakthrough curves of a tracer pulse is illustrated. This is done by comparing the results with those obtained from the exact analytical solution given in Eq. (42) and also from a numerical approach that inversely transforms the Laplace-transformed solution to the exact analytical solution back to the time domain with the use of the De Hoog algorithm (De Hoog et al. 1982). For comparison purposes, the $q_{\text {mean }}$ is varied in the range from 0.2 to $8.0 \mathrm{ml} / \mathrm{d}$.

As shown in Fig. 6, the exact analytical solution gives identical results with those obtained from the numerical solution, which is, however, expected. The comparisons in Fig. 6 also indicate that the agreement between the breakthrough curves calculated from the approximated solution and the exact analytical solution are satisfactory when $q_{\text {mean }}>1.0 \mathrm{ml} / \mathrm{d}$, corresponding to $u>0.05 \mathrm{~m} / \mathrm{d}$, provided that the fracture is conceptualized as a single flow path. This triggers an alert that, as discussed in the previous, the approximated solution given in Eq. (45) can only be used in the high flow-rate cases for non-sorbing solutes. However, it also suggests that the approximated solution might find more applications in the interpretation of field tracer tests because they are often performed at high flow rates, larger than, e.g. $15.0 \mathrm{ml} / \mathrm{d}$, in order to shorten the test period. In the following subsection, the different performance of the single-flow-path and multi- channel models are studied with regard to predicting the breakthrough curves of a tracer pulse.

\section{Comparison of the model predictions}

The fundamental difference between the single-flow-path and multi-channel models lies in the way hydrodynamic dispersion is dealt with. For channels with constant aperture, the single-flow-path model accounts only for Fickian dispersion, which acts only across the aperture of the flow channels in accordance with the classical Taylor dispersion theory (Taylor 1953; Bird et al. 2002). It may, however, be readily extended to account for channels with variable apertures, in which case much larger Fickian dispersion could result, as detailed in Appendix B. The multi-channel model considers both Fickian dispersion, which acts across the aperture or width of the flow channels with variable aperture, and velocity dispersion due to different flow rates of the channels. In tapered channels, which are deemed to better describe flow paths in fractured crystalline rocks, Taylor dispersion caused by diffusion across the aperture of the channels is negligible compared to that caused by diffusion across the width of the channels. Consider a tapered channel with $a_{0}=200 \mu \mathrm{m}$ and $W=0.1 \mathrm{~m}$, as illustrated in Appendix B. Provided $u=0.1 \mathrm{~m} / \mathrm{d}$, it can be shown that the dispersion coefficient $D_{\mathrm{f}}$ that accounts for the effect of diffusion across the aperture of the channel would be nearly the same as $D_{\mathrm{w}}=1.6 \times 10^{-9} \mathrm{~m}^{2} / \mathrm{s}$, according to Eq. (33), whereas the dispersion coefficient that accounts for the effect of diffusion across the width of the channel becomes $1.76 \times 10^{-7} \mathrm{~m}^{2} / \mathrm{s}$, as Eq. (34) predicts. It can be seen that the latter is generally much larger. Therefore, in the present paper, the authors will only consider Fickian dispersion caused by diffusion across the width of the channels, and use Eq. (34) to evaluate the dispersion coefficient.

In order to make the predicted results comparable in the two models, the single-flow-path model, Eq. (21), needs, then, only information on the shape and mean size of the channels.
Fig. 6 Breakthrough curves at $x=2 \mathrm{~m}$ for the flow rate $q_{\text {mean }}=0.2,1.0,2.0,5.0$ and $8.0 \mathrm{ml} / \mathrm{d}$ (from right to left), respectively

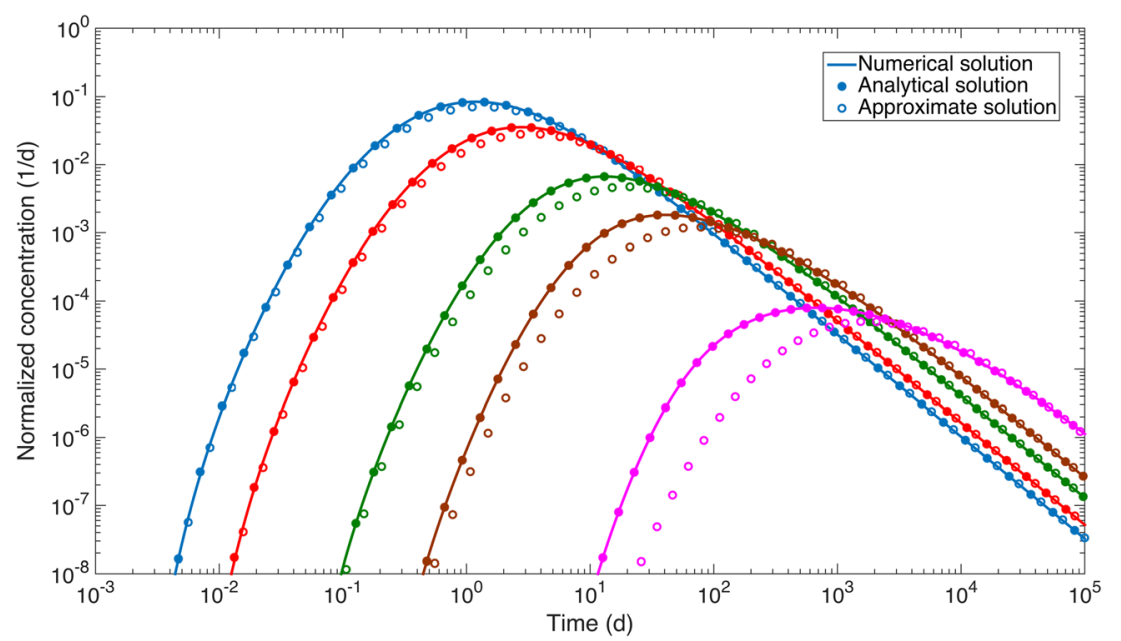


Together with the mean flow rate, this information is sufficient to determine the $f(t)$ function as well as the ratio between the flow volume and the flow-wetted-surface area, $b$. The multichannel model, Eq. (40), needs, in addition, to know the probability distribution function $\kappa(q)$ of the flow rates, which can be derived from the aperture distribution of the channels, as detailed in Appendix C.

Due to the inherent difference of the two models, the predictions as to how a tracer pulse spreads with increasing distance would be entirely different. As shown in Fig. 7, for the case of impermeable matrix, i.e. $\varepsilon_{\mathrm{p}}=0$, the breakthrough curves predicted by the single-flow-path and multi-channel models exhibit very different behaviors. In general, the results of the single-flow-path model resemble narrow spears with the extent of solute spreading slowly increasing with increasing distance. The mean $\bar{t}_{\mathrm{s}}$ and standard deviation $\sigma_{\mathrm{s}}$ of solute residence times, expressed as $\bar{t}_{\mathrm{s}}\left(\sigma_{\mathrm{s}}\right)$, are found to be 200 (34), 400 (48), and 800 (68) days, respectively, if observations are made at $x=2,4$, and $8 \mathrm{~m}$ from the origin of the fracture. Therefore, both $\bar{t}_{\mathrm{s}}$ and $\sigma_{\mathrm{s}}^{2}$ (i.e. the variance) increase in proportion to the distance the solute traveled. This leads to a linear increase of the Peclet number according to an expression analogous to Eq. (80) in Appendix C, a natural result of using a constant dispersion coefficient in the model. However, the calculated Pe values, varying from 145 to 580, are far larger than that observed in field tracer tests, which suggested that Pe is commonly in the range between 1 and 100 with a predominance of $\mathrm{Pe}=1 \sim 10$ (Gelhar et al. 1992; Gelhar 1993).

By contrast, the breakthrough curves of the multichannel model look more like bows skewed to the right with the skewness increasing significantly with the distance of observation. The mean $\bar{t}_{\mathrm{s}}$ and standard deviation $\sigma_{\mathrm{s}}$ of solute residence times shown in Fig. 7 are found to be 200 (98), 400 (188), and 800 (365) days, respectively, at a point of observation at $x=2,4$, and $8 \mathrm{~m}$. Compared to the results predicted by the single-flow-path model, the mean residence time $\bar{t}_{\mathrm{s}}$ is nearly identical but the variance $\sigma_{\mathrm{s}}^{2}$ becomes much larger in all of the three cases. More importantly, $\sigma_{\mathrm{s}}^{2}$ is now shown to increase in proportion to the traveling distance squared. This leads to the Peclet number unchanged, keeping roughly at 10.0, a typical value found in field tracer tests (Gelhar 1993). As a result, the dispersion coefficient $D_{\mathrm{f}}$ increases proportionally to the distance of observation, due primarily to the effect of velocity dispersion, which is consistent with a multitude of field observations (Gelhar et al. 1992; Gelhar 1993).

In the example illustrated in Fig. 7, the rock matrix is assumed to be impermeable in order to highlight the role of the two dispersion mechanisms. When matrix diffusion is also taken into consideration, the results shown in Fig. 8 suggest that matrix diffusion (Neretnieks 1980) causes strong retardation of solute transport, due to the great capacity of the porous rock in retaining the solute (Mahmoudzadeh et al. 2016), and therefore it dominates eventually over hydrodynamic dispersion in determining the spreading of a tracer pulse. Radioactive decay is, on the other hand, also an important factor decisive for the late tail of the breakthrough curves (Shahkarami et al. 2015); however, the general features shown in Fig. 7 as to the spreading of the tracer pulse are still kept in Fig. 8 -i.e. the multi-channel model predicts that the solute will arrive much earlier and that the variance of solute residence times will be much larger than what the single-flowpath model would predict.

\section{Extrapolation to other distances}

Due to the inherent difference of the single-flow-path and multi-channel models, they would also behave differently in extrapolating the experimental results of field tracer tests obtained at short distance to other distances. To illustrate this, a fictitious experiment is considered in the following. The experiment is performed at a flow rate of $q_{\text {mean }}=0.2 \mathrm{ml} / \mathrm{d}$ over a period of 100 years (in practice, field experiments are often performed at high flow rates over a period of, e.g. several
Fig. 7 Breakthrough curves at $x=2,4$ and $8 \mathrm{~m}$ (from left to right), respectively, predicted by the two models assuming $\varepsilon_{\mathrm{p}}=0$. The Fickian dispersion coefficient is determined by Taylor dispersion based on channel width. No decay is accounted for

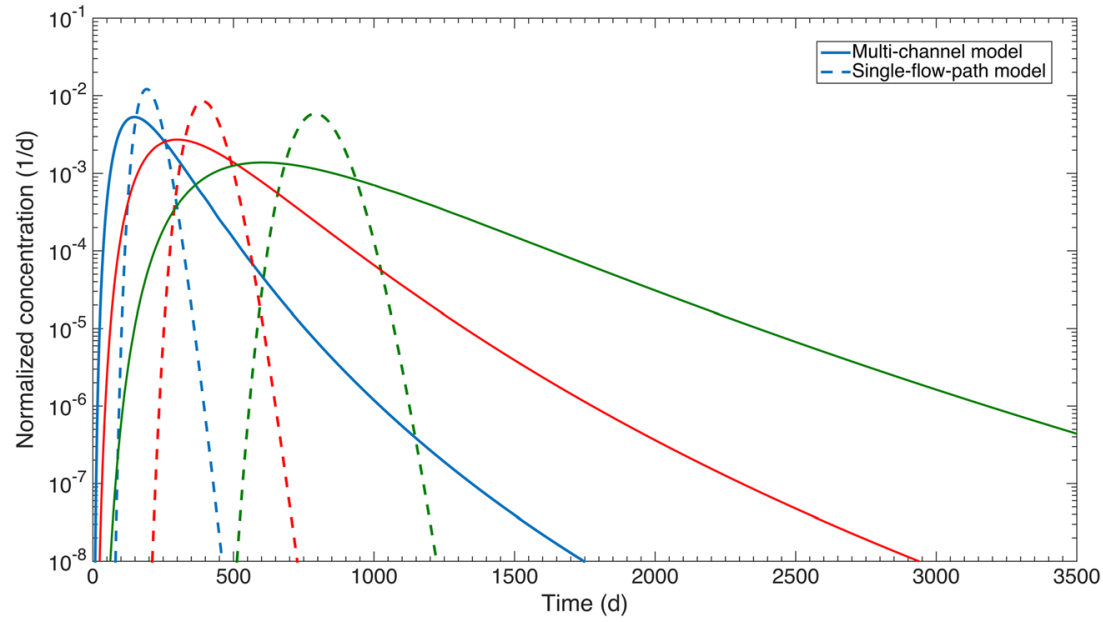


Fig. 8 Breakthrough curves at $x=2,4$ and $8 \mathrm{~m}$ (from left to right), respectively, predicted by the two models accounting for Fickian dispersion and matrix diffusion. The Fickian dispersion coefficient is determined by Taylor dispersion based on channel width

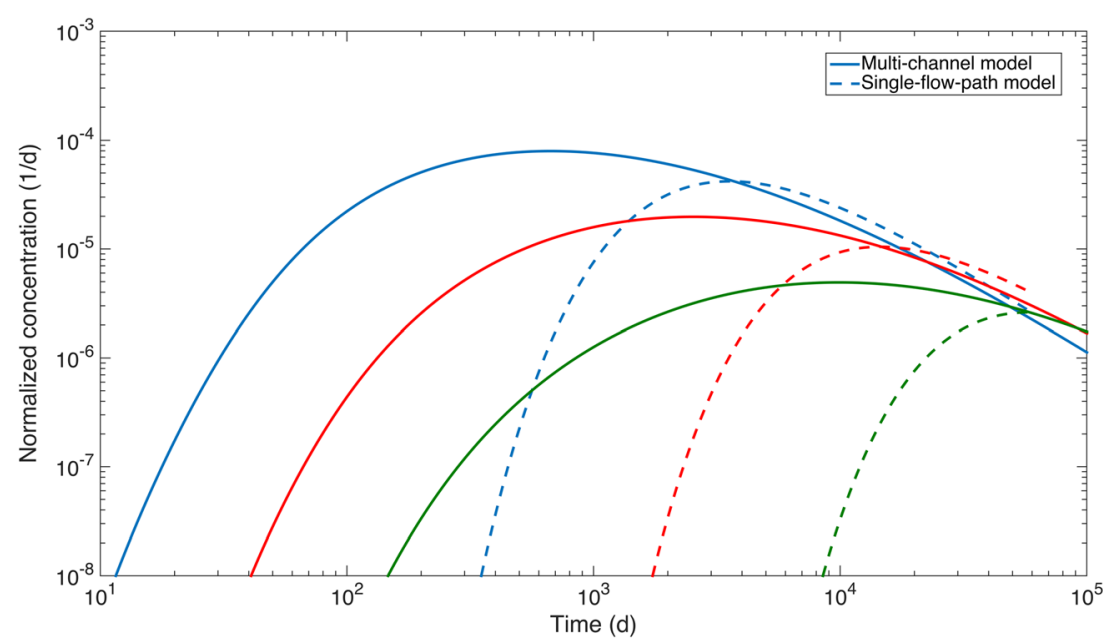

hundred days). The experiment data are generated from the simulation results of the multi-channel model perturbed with a random fluctuation so that the single-flow-path model can also reproduce the results. The breakthrough curve of a nonsorbing tracer obtained at the point of observation at $x=2 \mathrm{~m}$ is assumed to be represented by the solid circles in Fig. 9.

Then, it is possible for both models to get reasonably good curve fits of the results by adjusting the water velocity $u$ and the dispersion coefficient $D_{\mathrm{f}}$, in the single-flow-path model, and calibrating the channel width $W_{\text {mean }}$ and standard deviation $\sigma$ of the aperture field of the ensemble of channels in the multi-channel model. Provided that this is the case, as shown in Fig. 9, the two models would give very different breakthrough curves at $x=4$ and $8 \mathrm{~m}$, respectively. Essentially, the feature observed in Fig. 8 would still hold, i.e. the multichannel model would predict that the solute will arrive much earlier with a much larger variance of solute residence times than what the single-flow-path model might expect- this example clearly indicates that field experiments over a necessarily short distance cannot give information on what would take place over long distances unless it is known that the mass spreading was caused by only Fickian dispersion and that there was no contribution from velocity dispersion, or vice versa. The slight difference in form of the fitted curves is not sufficient to separate the impact of the two mechanisms. In other words, one should not feel satisfied with good curve fits of the experimental results unless the model and the parameters used are physically reasonable and have been verified independently. However, assuming $u=0.008 \mathrm{~m} / \mathrm{d}$ and $D_{\mathrm{f}}=$ $7.88 \times 10^{-8} \mathrm{~m}^{2} / \mathrm{s}$ have been used in the single-flow-path model for achieving good curve fits, one can reject its use in the predictions since the incredibly large $D_{\mathrm{f}}$ value is inconsistent with Taylor dispersion theory even for much wider channels then $W=0.1 \mathrm{~m}$ used in the example.

This analysis demonstrates the generality of the multichannel model, compared with the single-flow-path model, and also suggests that it is necessary to have a full understanding of how the performance of the multi-channel model would be influenced by a change of its parameters, which will be addressed in the following subsection.
Fig. 9 Using both models to fit the breakthrough curve observed at $x=2 \mathrm{~m}$ (left curves), and then extrapolating to the distances at $x=4$ and $8 \mathrm{~m}$. No decay is accounted for

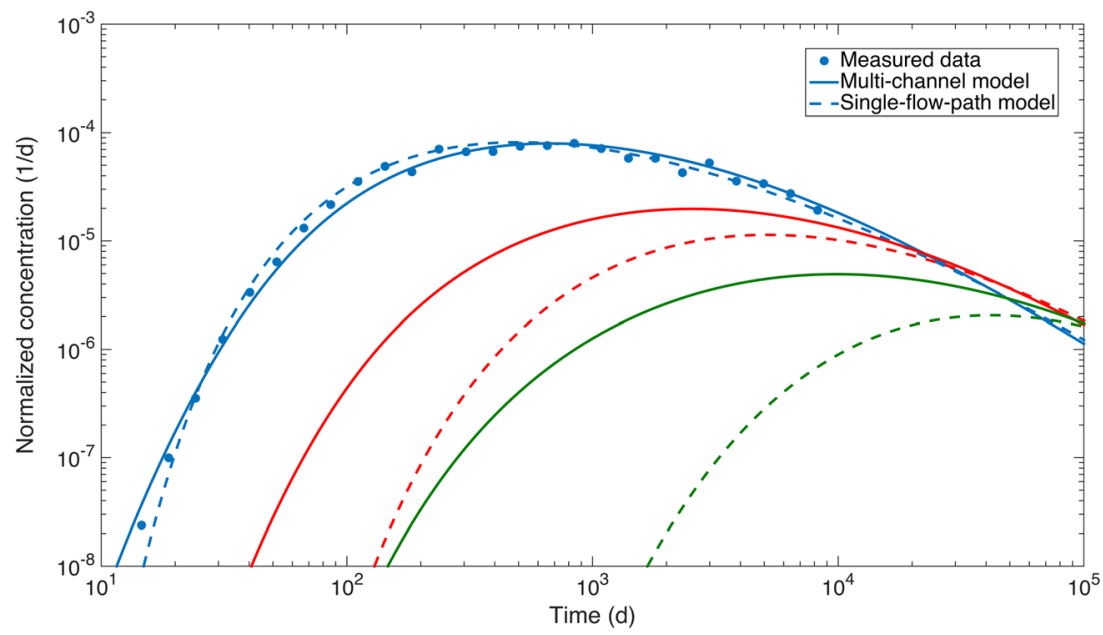




\section{Sensitivity analysis of the multi-channel model}

As discussed in Appendix C, the six key parameters in the multi-channel model can be classified into three categories: (1) the mean $a_{\text {mean }}$ and the standard deviation $\sigma$ of the aperture field that characterize the aperture variations of the ensemble of channels, for a given geometry of cross sections, (2) the mean half-width $W_{\text {mean }}$ and the $m$ parameter that describe the width variations of the ensemble of channels, (3) the mean flow rate $q_{\text {mean }}$ and the $n$ parameter that characterize the flow field of the ensemble of channels.

\section{Dependency on channels aperture mean and standard deviation}

For natural fractures, $a_{\text {mean }}$ can be obtained from highresolution imaging of fractures, i.e. computer aided tomography (CAT) X-ray scanning (Keller 1997) or other techniques (Hakami and Larsson 1996). It is often found to vary in the range between 0.1 and $1.0 \mathrm{~mm}$, and its influence on the calculated breakthrough curves is basically twofold. First, an increase in $a_{\text {mean }}$ would decrease the water velocity $u$ in each of the channels, and therefore would also cause a decrease in Fickian dispersion by decreasing $D_{\mathrm{f}}$ in Eq. (34). The net effect is to change the $f(t)$ function for each of the channels, making it more narrow shaped, leading to later arrival of the solute and a sharper increase in the concentration, as shown in Fig. 10. Second, an increase in $a_{\text {mean }}$ would also increase the mean half-aperture $b_{\text {mean }}$, as suggested by Eq. (44), which is equivalent to a decrease in the flow-wetted-surface area and hence changes the $g(t)$ function for each of the channels, making the late tail of the breakthrough curves less dependent on the change of $a_{\text {mean }}$, as seen in Fig. 10.

The standard deviation $\sigma$ can also be obtained from mapping of aperture of overcored fractures (Hakami and Larsson 1996; Keller 1997). It may vary from 0.07 to 0.50 according to Eq. (82), for the Pe values typically observed in field experiments. An increase in $\sigma$ would only change the $f(t)$ function for each of the channels, making the majority of flow to occur in the wider channels. This leads to a stronger velocity-dispersion, and therefore an earlier arrival of the solute with a much larger variance of solute residence times, as shown in Fig. 11.

It is to be noted that, in the assessment of hydraulic tests in boreholes (Neretnieks and Moreno 2003; Crawford et al. 2003), the mean $a_{\text {mean }}$ and the standard deviation $\sigma$ of the aperture field can be related to the flow rate distributions via the cubic law. It is, therefore, possible to estimate the probability distribution function of the volumetric flow rate of the channels $\kappa(q)$ in two independent ways.

\section{Dependency on channel width and the mean flow rate}

Up until now, there is little data on the width distributions and on the correlation between the width and the aperture of channels. Nevertheless, observations in drifts and tunnels suggested that the channel widths commonly vary from a few millimeters to several tens of centimeters (Tsang and Neretnieks 1998). They may be expected to increase with increasing aperture due to the correlations of spatial variations of the aperture field (Liu and Neretnieks 2006). For simplicity, $m$ may be considered $m=0$ or 1 . In any case, as shown in Fig. $12, W_{\text {mean }}$ only influences the $f(t)$ function. An increase in $W_{\text {mean }}$ would lead to a significant increase in $D_{\mathrm{f}}$ in Eq. (34), leading to a larger Fickian dispersion, and also a stronger velocity-dispersion due to an increase in the degree of variations of water velocities. A linear relation between the half-width and the aperture, i.e. $m=1$, would make these effects more prominent.

The mean flow rate $q_{\text {mean }}$ is, on the other hand, always known in field tracer tests, and for deep repositories in crystalline rocks it can be estimated from the fact that the groundwater velocity may span a range between 1 and 1,000 m/year. The effect of $q_{\text {mean }}$ on the predicted breakthrough curves is
Fig. 10 Dependence of the breakthrough curves of the multichannel model on the mean aperture of the ensemble of channels

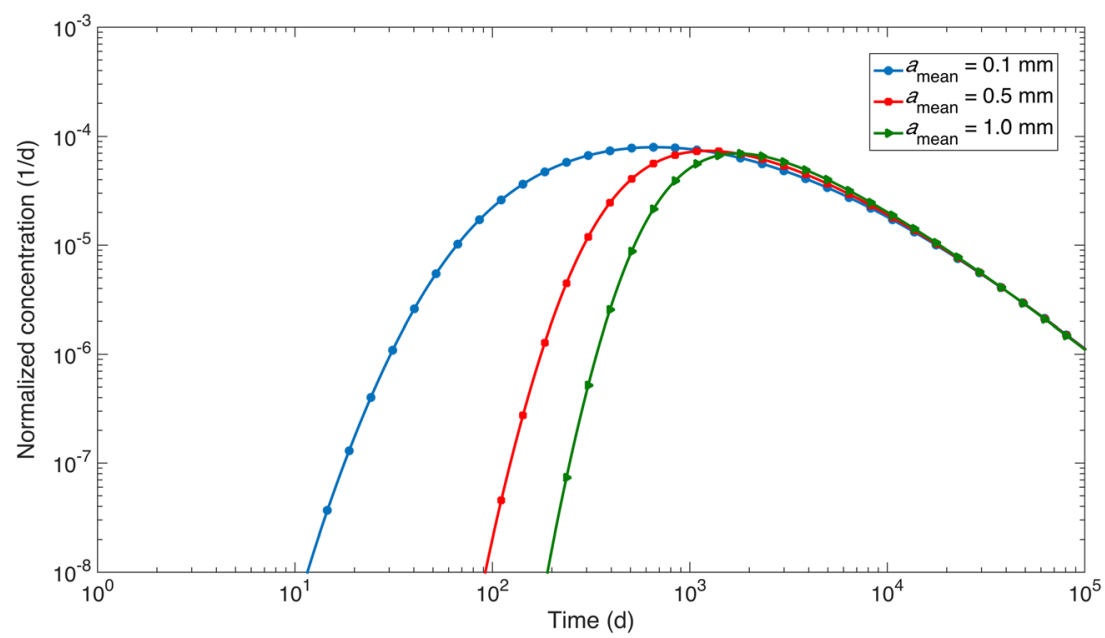


Fig. 11 Dependence of the breakthrough curves of the multichannel model on the standard deviation of the apertures of the ensemble of channels

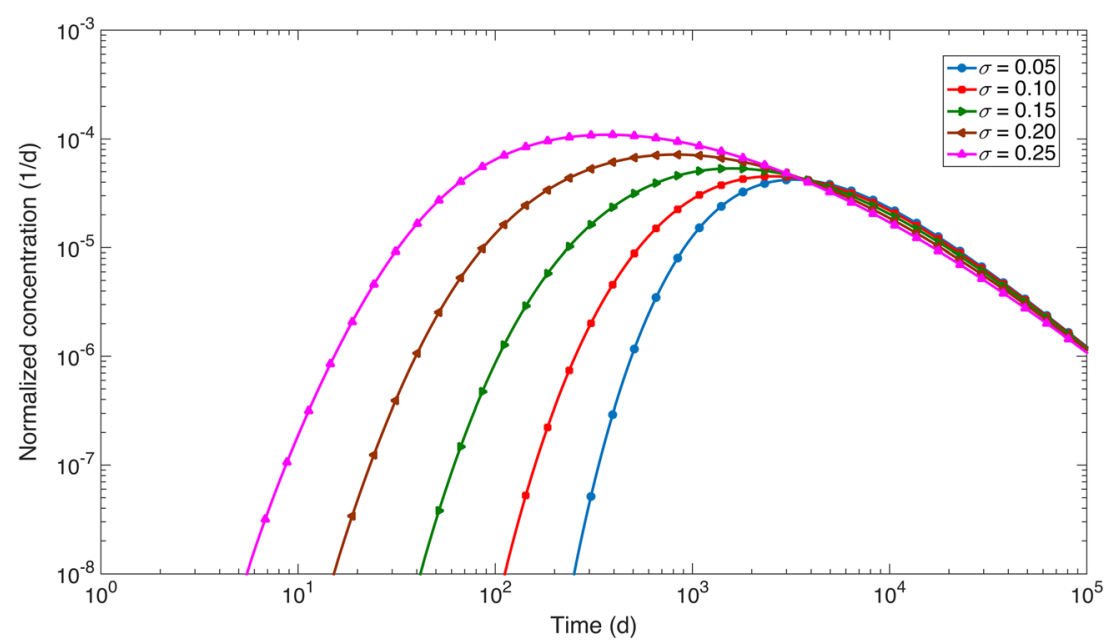

also twofold, similar to that of $a_{\text {mean }}$. First, an increase in $q_{\text {mean }}$ would obviously increase water velocity $u$ in all of the channels, and therefore both Fickian and velocity dispersion would become stronger. As a result of this effect on changing the $f(t)$ function, the solute will arrive much earlier with a much wider spreading of solute mass in the early time breakthrough curves, as shown in Fig. 13. Second, an increase in $q_{\text {mean }}$ would make the ratio between the flow-wetted-surface area and the flow rate of each of the channels decrease, weakening the exchange between the fracture and the rock matrix (Neretnieks 1980, 2002). Hence, the impact of the change on the $g(t)$ function, which contains the information of FWS/ $q$, leads to an earlier release of solute from the rock matrix, as seen in Fig. 13.

The $n$ parameter used to describe the correlation between the mean water velocity $u$ and the mean aperture, as given in Eq. (72), is commonly taken to be 2 by following the cubic law of laminar flow in a slit (Bird et al. 2002; Neretnieks 2002). Mainly for illustrative purpose, its influence on the behaviors of the breakthrough curves is also investigated by keeping $\sigma=0.2135$, the value obtained at $\mathrm{Pe}=10$ and $n=2$, via Eq. (82).
As shown in Fig. 14, an increase in the $n$ value significantly enlarges both Fickian dispersion, by way of an increase in $D_{\mathrm{f}}$ in Eq. (34), and velocity dispersion, by virtue of a large spreading of the water velocities. Obviously, its effect on changing the $f(t)$ function is similar to that caused by the standard deviation $\sigma$ of apertures of the ensemble of channels, see Eq. (81) and also Fig. 11.

The analysis so far concerns only tapered channels, as schematically shown in Appendix B. If one assumes the fracture is composed of sinusoidal wave-shaped channels instead, as also shown in Appendix $\mathrm{B}$, the only change is the $C_{\mathrm{D}}$ value used in evaluating the dispersion coefficient $D_{\mathrm{f}}$, which changes from 48 to 77.9. This would result in a less Fickian dispersion, as shown in Fig. 15, provided that the other parameters are kept unvaried. The effect is, then, equivalent to a slight decrease in $W_{\text {mean }}$ (by a factor of $\sim 0.8$ ), while still assuming that all channels are of tapered type with rhomboidal cross section, which suggests that the shape of the channels does not really matter in affecting the behavior of the multi-channel model.

Undoubtedly, the multi-channel model is more general and more competitive than the single-flow-path model in
Fig. 12 Dependence of the breakthrough curves of the multichannel model on channel width, for $m=0$ (solid line) and 1 (dashed line), respectively

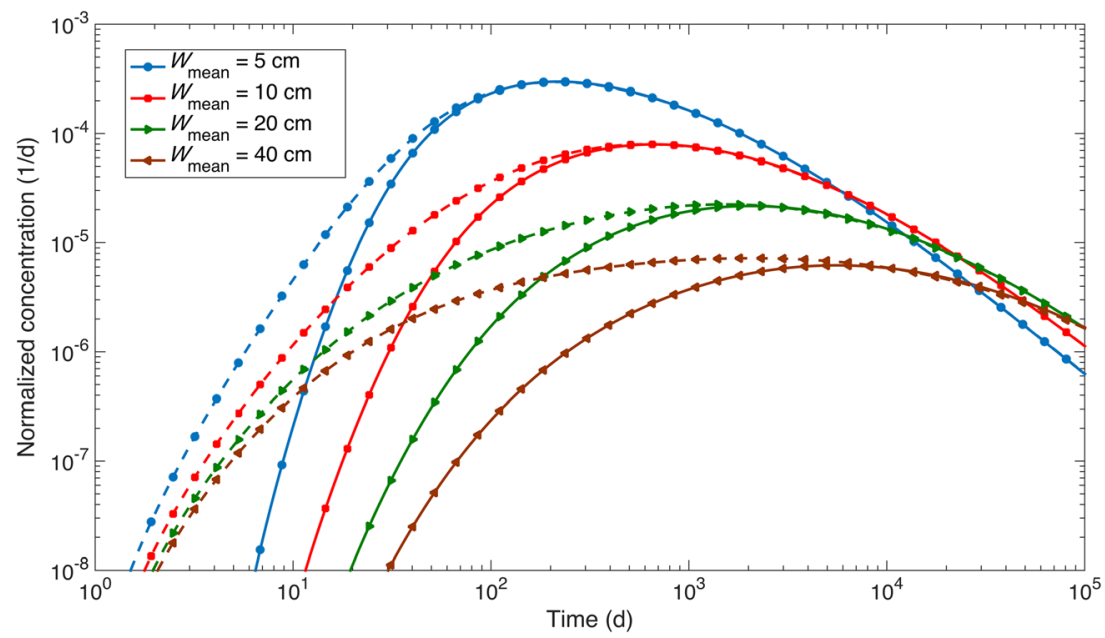


Fig. 13 Dependence of the breakthrough curves of the multichannel model on the mean flow rate of the ensemble of channels

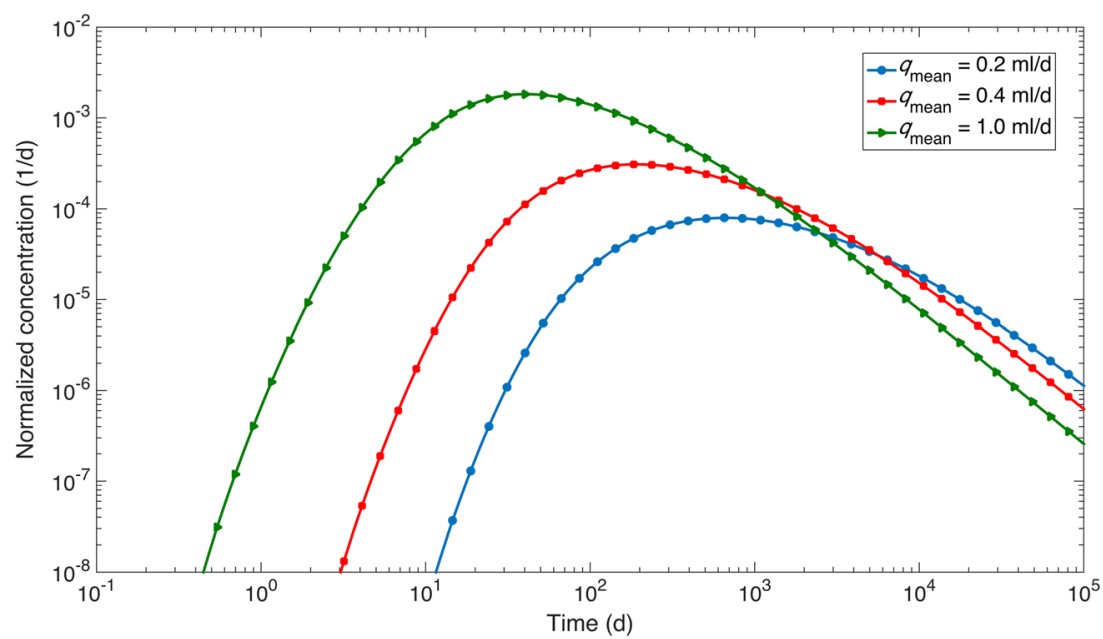

predicting solute transport in fractured rocks by accounting for both Fickian and velocity dispersions, albeit a bit complex. There is, however, still a long way to go before implementing it into, e.g. the channel network model (Gylling et al. 1999) for safety and performance assessment of deep geological repositories for spent nuclear fuel. In particular, the capability and malleability of the multi-channel model in interpreting fieldscale experiments should be extensively studied in the near future.

\section{Conclusions}

In this contribution, the model proposed by Tang et al. (1981) is revisited and a new simple and robust solution is developed for the problem of solute transport along a single fracture in a porous rock. This is called the solution to the single-flow-path model. Similar to the analytical solution of Tang et al. (1981), the new solution, as given in Eq. (21), takes the form of an integral. However, the solution now becomes an apparent convolution of two functions, the $f(t)$ and $g(t)$ functions, with finite limits of integration. This makes the numerical integration much more stable and efficient.

The $f(t)$ function is the impulse response of the fractureonly system. Therefore, it describes the probability density function of the residence time distribution of a conservative solute resulting merely from advection and dispersion. The $g(t)$ function is the response of the fracture-matrix system to the $c_{\text {in }}(t)$ injection in the limiting case when $\mathrm{Pe} \rightarrow \infty$, i.e. $D_{\mathrm{f}} \rightarrow 0$, given a solute-advection time (the distribution of which is determined by the $f(t)$ function). It characterizes the mass exchange between the fracture and the rock matrix. As a result, the effects of Fickian dispersion and matrix diffusion on solute transport have been apparently decoupled, which allows for these two transport mechanisms to be considered separately and independently.

Noticeably, the $f(t)$ function for solute residence time distribution of a fracture-only system can also be understood as a result of the solute having fictitiously followed streamlines with different constant velocities. No diffusion between these streamlines and no diffusion/dispersion in the flow direction in a streamline is, however, invoked because the effect of Fickian dispersion has already been taken into account by
Fig. 14 Dependence of the breakthrough curves of the multichannel model on the $n$ parameter characterizing the water velocity

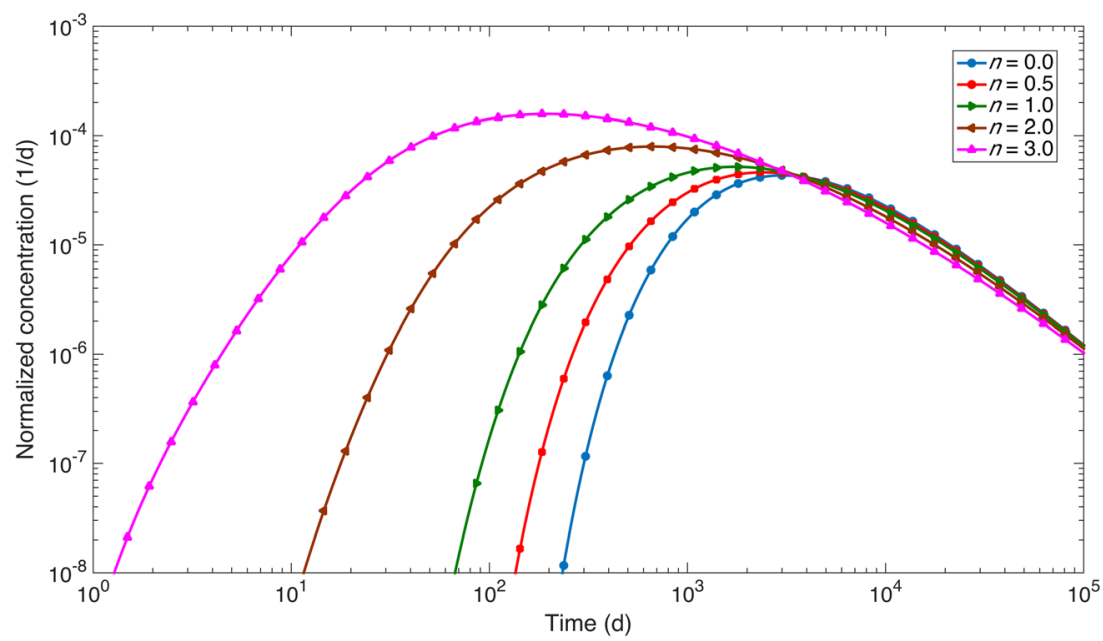


Fig. 15 Dependence of the breakthrough curves of the multichannel model on channel geometry

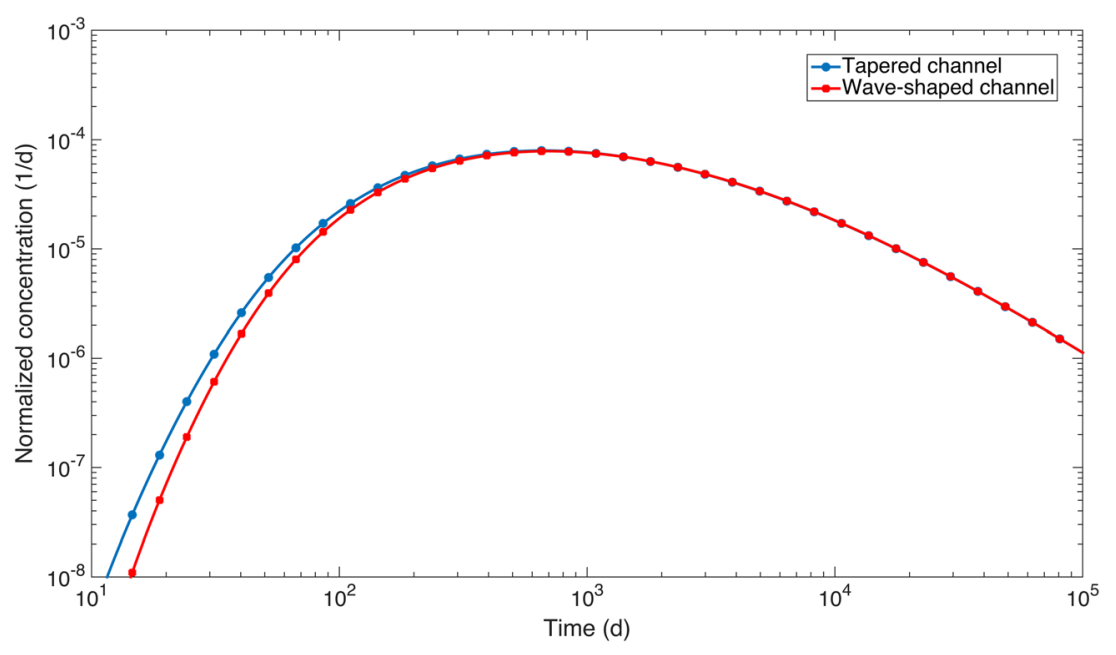

the distribution of solute-advection time along the streamlines. As described by the $g(t)$ function, the dispersion of the solute along each fictitious stream tube is now caused only by matrix diffusion. The collecting and mixing of the solutes at the outlet of different stream tubes gives, then, the concentration of $c_{\mathrm{f}}(t)$, as explained in Fig. 3. This interpretation of the new solution is well in line with the concept of velocity dispersion (Neretnieks 1983), making it possible to treat both Fickian and velocity dispersions by the same formalism and therefore allows for a simultaneous accounting for these two dispersion mechanisms.

It follows that the new simple solutions obtained from the Fickian dispersion case can be extended into more general cases where a discrete/continuous set of flow channels with different flow rates is present in fractures in rocks. This leads to a development of the multi-channel model in contrast to the single-flow-path model. The difference between the two models lies basically in how to deal with hydrodynamic dispersion. The single-flow-path model accounts only for Fickian dispersion due to diffusion across the aperture of the channels as well as across the width. The latter gives rise to much Fickian dispersion. The multi-channel model considers, however, not only Fickian dispersion but also velocity dispersion, resulting from different flow rates of the channels.

As a result, the exact analytical solution for the multichannel model given in Eq. (42), normally cannot take the same simple form as that of the solution to the single-flowpath model; nevertheless, it is shown that for non-sorbing solutes, a good approximation can be made in the high flowrate cases to give an approximated solution. This solution, as given in Eq. (45), takes the same form as that of the solution to the single-flow-path model and is expected to find more applications in the interpretation of field tracer tests because they are often performed at high flow rates over a short period.

The simulations based upon a representative case with slowly seeping water in fractured rocks suggest that the use of the single-flow-path model for practical applications is questionable if Fickian dispersion is taken into account only for diffusion across the channel apertures by the classical Taylor dispersion theory (Taylor 1953; Bird et al. 2002). For diffusion across the channel width, the Fickian dispersion can also be overshadowed by velocity dispersion at longer travel distances. This is also supported by a multitude of field observations (Gelhar et al. 1992; Gelhar 1993; Hjerne et al. 2010), which suggested that the Peclet number stays basically unchanged with the distance and often lying in the range between 1 and 100. By contrast, the predictions of the multichannel model agree well with what was observed in the field, by accounting for both Fickian and velocity dispersions.

In addition, it is found that the single-flow-path and the multi-channel models behave very differently in extrapolating the results of field tracer tests to other distances, which suggests that short-range field experiments cannot be used to determine dispersion over large distances if based only on the classical single-flow-path model. The generality of the multi-channel model, which incorporates the single-flowpath model, makes it, however, more suitable for the interpretation of field tracer tests because it can readily be used in understanding and modeling the effects of both Fickian and velocity dispersions on solute transport in fractured rocks.

Finally, to provide a more in-depth insight into the multichannel model, a sensitivity analysis is performed to assess the relative contributions of the model key parameters to the overall dispersion, i.e. broadening due to the hydrodynamic dispersion and matrix diffusion. In general, the results revealed a strong dependence of the model on variations in properties of the ensemble of channels, i.e. channels aperture, width and flow-rate distribution, though the effect of channel shape was negligible.

Additionally, it should be noted that the multichannel model can also be extended to include different transport and interaction mechanisms along different flow channels such as heterogeneity in a porous rock (Haggerty and Gorelick 1995; Haggerty 1999). This, 
together with its implementation into, e.g. the channel network model (Gylling et al. 1999), will be made in the near future.

Acknowledgements The authors gratefully acknowledge the encouragement and financial support of the Swedish Nuclear Fuel and Waste Management Company (SKB).

\section{Appendix A: Analytical solutions in a limiting case}

For ease of reference, some analytical solutions in the limiting case as $\mathrm{Pe} \rightarrow \infty$, i.e. $D_{\mathrm{f}} \rightarrow 0$, are presented here.

In the case of a Dirac delta injection, $c_{\text {in }}$ can be written

$c_{\text {in }}=m_{0} \delta(t)$

where $m_{0}$ is the ratio between the mass injected and the volumetric flow rate through the fracture.

The solution to $c_{\mathrm{f}}$ is, then, given by

$\left.c_{\mathrm{f}}\right|_{\mathrm{Pe} \rightarrow \infty}=m_{0} \exp (-\lambda t) \alpha\left(t-R_{\mathrm{f}} t_{\mathrm{w}}, G t_{\mathrm{w}}\right)$

with

$\alpha(t, A)=\frac{A}{2 \sqrt{\pi t^{3}}} \exp \left(-\frac{A^{2}}{4 t}\right) H(t)$

where $H(t)$ is the Heaviside step function.

In the case of a Heaviside step injection, $c_{\text {in }}$ can be written

$c_{\text {in }}=c_{0} H(t)$

where $c_{0}$ is the source concentration.

The solution to $c_{\mathrm{f}}$ then becomes

$\left.c_{\mathrm{f}}\right|_{\mathrm{Pe} \rightarrow \infty}=\frac{c_{0}}{2} \exp \left(-\lambda R_{\mathrm{f}} t_{\mathrm{w}}\right) \beta\left(t-R_{\mathrm{f}} t_{\mathrm{w}}, G t_{\mathrm{w}}\right)$

With

$$
\begin{aligned}
\beta(t, A) & =\exp (-A \sqrt{\lambda}) \operatorname{erfc}\left(\frac{A}{2 \sqrt{t}}-\sqrt{\lambda t}\right) H(t) \\
& +\exp (A \sqrt{\lambda}) \operatorname{erfc}\left(\frac{A}{2 \sqrt{t}}+\sqrt{\lambda t}\right) H(t)
\end{aligned}
$$

In the case when the injection over a time period of $\Delta t$ is defined by a decaying top-hat function, as given in Eq. (27), the solution to $c_{\mathrm{f}}$ can be written as

$\left.c_{\mathrm{f}}\right|_{\mathrm{Pe} \rightarrow \infty}=c_{0} \exp (-\lambda t)\left[\gamma\left(t-R_{\mathrm{f}} t_{\mathrm{w}}, G t_{\mathrm{W}}\right)-\gamma\left(t-R_{\mathrm{f}} t_{\mathrm{w}}-\Delta t, G t_{\mathrm{W}}\right)\right]$

with

$\gamma(t, A)=\operatorname{erfc}\left(\frac{A}{2 \sqrt{t}}\right) H(t)$
In general cases, the solution to $c_{\mathrm{f}}$ takes the form of a convolution integral, i.e.

$\left.c_{\mathrm{f}}\right|_{\mathrm{Pe} \rightarrow \infty}=\left.\int_{0}^{t} c_{\mathrm{in}}(t-\tau) c_{\mathrm{f}}(\tau)\right|_{\delta, \mathrm{Pe} \rightarrow \infty} \mathrm{d} \tau$

where the subscript $\delta$ refers to the case of a Dirac delta injection, and therefore the term with this subscript is the impulse response defined by Eqs. (47) and (48) with $m_{0}$ taken to be unity.

It follows that the solution in its general form can be written as

$\left.c_{\mathrm{f}}\right|_{\mathrm{Pe} \rightarrow \infty}=g\left(t-R_{\mathrm{f}} t_{\mathrm{w}}, G t_{\mathrm{w}}\right) H\left(t-R_{\mathrm{f}} t_{\mathrm{w}}\right)$

which is the origin of Eq. (13).

\section{Appendix B: Taylor dispersion in channels and transverse mixing along a flow path}

Solute carried along streamlines with different velocities diffuses not only along the streamlines but also between them. In narrow channels, the molecular diffusion across the streamlines can rapidly even out the concentration profile that would result from the different velocities. Taylor (1953) showed that in a circular tube where the velocity profile is parabolic, the spreading of a tracer pulse, which would become infinitely drawn out if there were no molecular diffusion, propagates similarly to a Gaussian pulse after an initial distance. The pulse travels with the mean fluid velocity and becomes broader in proportion to the square root of the travel distance (or the travel time) exactly as a pulse would with a larger molecular diffusion coefficient. In other words, the effect of dispersion can be described by a constant dispersion coefficient that is much larger than the molecular diffusion coefficient $D_{\mathrm{w}}$. For a long, straight tube of radius $R$, it can be calculated by Taylor (1953)

$D_{\mathrm{f}}=D_{\mathrm{w}}+\frac{u^{2} R^{2}}{48 D_{\mathrm{w}}}$

For a narrow slot with an aperture $2 b$, the constant changes to 52.5 with $b$ in place of $R$ (Bird et al. 2002), as given in Eq. (33). With this brief discussion, the authors may now consider a similar effect of diffusion between streamlines in a tapered channel in a fracture, as schematically shown in Fig. 16. Due to the geometric symmetry, it is only needed to study the right half of the channel; the local aperture, $a$, decreases linearly as $y$ increases, i.e.

$a=a_{0}\left(1-\frac{y}{W}\right)$

where $W$ is the half width of the channel, and $a_{0}$ is the largest aperture of the channel located at $y=0$. 


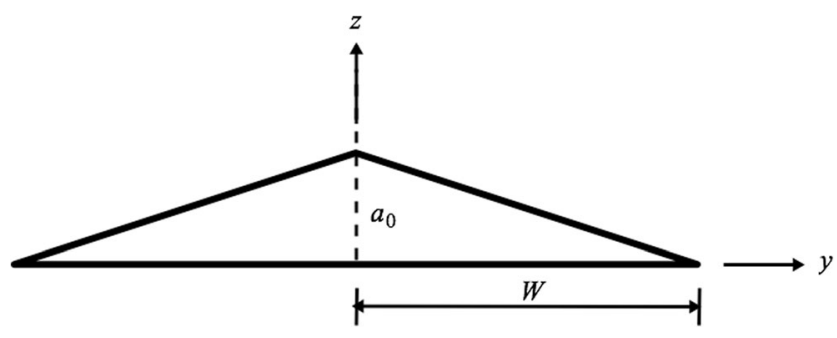

Fig. 16 Cross section of a tapered channel, in which the velocity is proportional to the local aperture. The flow is directed into the plane of the figure

Correspondingly, the local flow velocity, $u_{\mathrm{x}}$, is assumed to be proportional to the local aperture squared; thus, it can be written

$u_{\mathrm{x}}=2 u\left(\frac{a}{a_{0}}\right)^{2}$

where the subscript $\mathrm{x}$ denotes the $\mathrm{x}$ direction and $u$ is the mean water velocity.

Since the aperture $a$ of the channel is much smaller than its width $W$, it is plausible to assume that molecular diffusion rapidly evens out the concentration across the aperture. Then, the advection-diffusion equation describing the concentration evolution in time and space takes the form

$\frac{\partial c}{\partial t}+u_{\mathrm{x}} \frac{\partial c}{\partial x}=D_{\mathrm{w}}\left(\frac{\partial^{2} c}{\partial x^{2}}+\frac{1}{a} \frac{\partial}{\partial y}\left(a \frac{\partial c}{\partial y}\right)\right)$

By substituting the expressions for $a$ and $u_{\mathrm{x}}$ in Eqs. (58) and (59), respectively, into Eq. (60), it can be obtained by defining $r=W-y$,

$\frac{\partial c}{\partial t}+2 u\left(\frac{r}{W}\right)^{2} \frac{\partial c}{\partial x}=D_{\mathrm{w}}\left(\frac{\partial^{2} c}{\partial x^{2}}+\frac{1}{r} \frac{\partial}{\partial r}\left(r \frac{\partial c}{\partial r}\right)\right)$

This equation is identical to that obtained by Taylor (1953) for a long, straight tube of radius $R$. The velocity distribution

$u_{\mathrm{x}}=2 u\left(\frac{r}{W}\right)^{2}$

is also parabolic, with the highest velocity at $y=0$ and the lowest at $y=W$.

Using the same methodology as Taylor (1953) to derive "Taylor dispersion" for this specific case, somewhat surprisingly, identical results are obtained for dispersion in a circular tube. The only difference is that the tube radius is exchanged for the half width of the channel, i.e.

$D_{\mathrm{f}}=D_{\mathrm{w}}+\frac{u^{2} W^{2}}{48 D_{\mathrm{w}}}$

which is the origin of Eq. (34).

To test the sensitivity of $D_{\mathrm{f}}$ to the geometry, a wave-shaped channel in a fracture as schematically shown in Fig. 17 is also

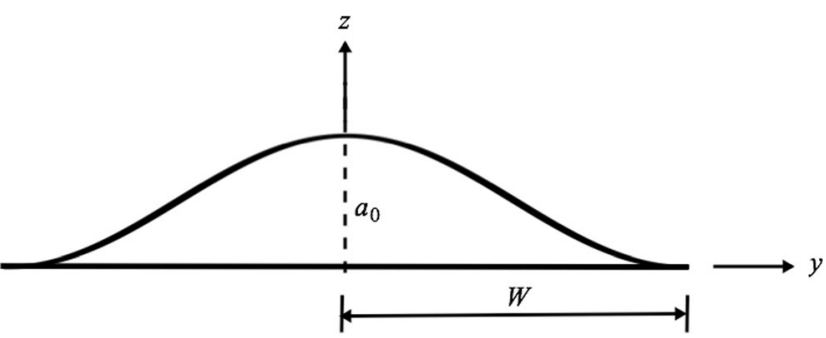

Fig. 17 Cross section of a wave-shaped channel, in which the velocity is proportional to the local aperture. The flow is directed into the plane of the figure

considered. The change of the local aperture of this channel is described by

$a=\frac{a_{0}}{2}\left[1+\cos \left(\frac{\pi y}{W}\right)\right]$

The result shows that the constant in Eq. (63) then becomes 77.9 , and as expected the dispersion is less in this case because the majority of flow occurs in the wider section. One can estimate the condition when the cross diffusion has had time (or traveled a sufficient distance) for dispersion of Taylor type to have stabilized by comparing the characteristic diffusion time $t_{\mathrm{D}}=W^{2} / D_{\mathrm{w}}$ with the advection time $t_{\mathrm{w}}=x / u$. The Taylor dispersion would be fully developed when $t_{\mathrm{D}} \ll 4 t_{\mathrm{w}}$ or when $W u / D_{\mathrm{w}} \gg 1$ (Wang et al. 2012). Under this condition, the advection-dispersion equation with a constant $D_{\mathrm{f}}$ at all distances would become valid, i.e.

$\frac{\partial c}{\partial t}+u \frac{\partial c}{\partial x}=D_{\mathrm{f}} \frac{\partial^{2} c}{\partial x^{2}}$

where $c$ should be understood as the mean concentration over the cross section of the channel. It should again be emphasized that $D_{\mathrm{f}}$ is constant at all distances. This is in contrast to practically all field observations, which indicates that $D_{\mathrm{f}}$ increases with the distance (Gelhar et al. 1992).

\section{Appendix C: characterization of flow channels in natural fractures}

When applying the solution in Eq. (21) into cases where a continuous set of channels with different flow rates is present in fractures in rocks, the following information is required to account for the effect of velocity dispersion: (1) the width distribution, (2) the relation between the volumetric flow rate $q$ and the half-aperture $b$ of the channels, (3) the relation between the water velocity $u$ and $b$, for evaluating the $f(t)$ function. Field observations suggest that, in general, wider channels tend to have larger apertures, i.e. they are correlated. Although channel widths and channel apertures may also have independent probability distributions, in the following, it is assumed that the probability density function of the aperture distribution can be determined independently and that the width is directly correlated to the aperture. From this the $f(t)$ 
function as well as the distribution of the specific flow-wettedsurface area of the flow channels, which determines the mass exchange between the fracture and the rock matrix, can be derived.

Specifically, one may characterize each of the channels with a half-width $W$ and a half-aperture $b$, through which water flows with a mean velocity $u$, as shown in Fig. 1 . The half-aperture $b$ should, however, be understood as the ratio between the flow volume, FLV, and the flow-wetted-surface area, FWS, of each of the flow channels, through which matrix diffusion occurs (Mahmoudzadeh et al. 2016), i.e.

$b=\frac{\mathrm{FLV}}{\mathrm{FWS}}$

depending, therefore, on the geometry of the channels, the aperture of which may vary across the width in, e.g. rhomboidal or wave-shaped channels.

The mean velocity $u$ is, on the other hand, the ratio between the volumetric flow rate $q$ and the cross-sectional area $A$ of each of the channels. It is an average of the local water velocities, which vary with the local aperture. In this study, the cubic law of laminar flow in a slit (Bird et al. 2002) is generalized to relate the local water velocity $u_{\mathrm{x}}$ to the local aperture $a$ by

$u_{\mathrm{x}}=k_{n} a^{n}$

where $k_{n}$ and $n$ are two constants characterizing the water velocity, and $n$ is equal to 2 in the cubic law case. This expression implies that there is negligible interference between the nearby streamlines by friction in the channel that is very wide compared to its aperture.

Moreover, numerous observations (Hakami and Larsson 1996; Keller 1997; Liu and Neretnieks 2006) suggested that the aperture field of natural fractures follows, mostly, a lognormal distribution without regard to horizontal spatial positions. It is, then, possible to postulate that the mean aperture $\bar{a}$ of the channels could be reasonably well described by a lognormal distribution function, i.e.

$p(\bar{a})=\frac{1}{\bar{a} \sigma \sqrt{2 \pi}} \exp \left[-\frac{(\ln \bar{a}-\mu)^{2}}{2 \sigma^{2}}\right]$

where $\mu$ and $\sigma$ are the mean and standard deviation of $\ln \bar{a}$, respectively.

On the other hand, the half-width $W$ of the channel may be independent of $\bar{a}$ in reality, but for now it is assumed that there is a relation between $W$ and $\bar{a}$ in the form

$W=k_{m} \bar{a}^{m}$

where $k_{m}$ and $m$ are two constants characterizing the channel width, and $m$ is equal to 0 when all channels have the same width.

With these postulations, the authors first consider the conventional way of describing the flow rate distributions in the channels in which they are assumed to have constant aperture. The approach is then generalized to consider the channels with varying aperture across the width, which aims to introduce the effect of Taylor-like dispersion, which acts in channels with constant aperture only across the aperture but not across the width of the channel, as discussed in Appendix B.

In the case where the fracture is assumed to consist of rectangular channels, the local aperture $a$ is identical to the mean aperture $\bar{a}$ everywhere in each of the channels. It is, then, straightforward to write for each of the channels,

$b=C_{\mathrm{s}}\left(\frac{\bar{a}}{2}\right)$

and the volumetric flow rate by

$q=2 C_{\mathrm{q}} k_{m} k_{n} \bar{a}^{m+n+1}$

where $C_{\mathrm{s}}$ and $C_{\mathrm{q}}$ are two factors accounting for the influence of the channel geometry on the flow conditions, and for rectangular channels $C_{\mathrm{s}}=C_{\mathrm{q}}=1$.

Correspondingly, the mean water velocity is given for each of the channels by

$u=C_{\mathrm{q}} k_{n} \bar{a}^{n}$

and, therefore, it can be obtained for the water residence time

$t_{\mathrm{w}}=\frac{L}{C_{\mathrm{q}} k_{n}} \bar{a}^{-n}$

where $L$ is the length of the channel.

As a result, the mean aperture of the ensemble of channels is given by

$a_{\text {mean }}=\int_{0}^{\infty} \bar{a} p(\bar{a}) \mathrm{d} \bar{a}=\exp \left(\frac{2 \mu+\sigma^{2}}{2}\right)$

and the mean half-width of the ensemble of channels can be expressed as

$W_{\text {mean }}=\int_{0}^{\infty} W p(\bar{a}) \mathrm{d} \bar{a}=k_{m} \exp \left(\frac{2 m \mu+m^{2} \sigma^{2}}{2}\right)$

Likewise, the mean flow rate of the ensemble of channels $q_{\text {mean }}$ can be written as

$q_{\text {mean }}=\int_{0}^{\infty} q p(\bar{a}) \mathrm{d} \bar{a}=2 C_{\mathrm{q}} k_{m} k_{n} \exp \left[\frac{2(m+n+1) \mu+(m+n+1)^{2} \sigma^{2}}{2}\right]$

and the mean water residence time as

$t_{\mathrm{w}, \text { mean }}=\int_{0}^{\infty} t_{\mathrm{w}} p(\bar{a}) \mathrm{d} \bar{a}=\frac{L}{C_{\mathrm{q}} k_{n}} \exp \left(\frac{-2 n \mu+n^{2} \sigma^{2}}{2}\right)$ 
The variance of the water residence time $t_{\mathrm{w}}$ can also be obtained from the second moment

$\sigma_{\mathrm{t}}^{2}=\int_{0}^{\infty} t_{\mathrm{w}}^{2} p(\bar{a}) \mathrm{d} \bar{a}-t_{\mathrm{w}, \text { mean }}^{2}$

The result is

$\sigma_{\mathrm{t}}^{2}=\left(\frac{L}{C_{\mathrm{q}} k_{n}}\right)^{2}\left[\exp \left(-2 n \mu+2 n^{2} \sigma^{2}\right)-\exp \left(-2 n \mu+n^{2} \sigma^{2}\right)\right]$

Following the discussion of Neretnieks (2002), it can then be found that the Peclet number

$\mathrm{Pe}=2\left(\frac{t_{\mathrm{w}, \text { mean }}}{\sigma_{\mathrm{t}}}\right)^{2}$

becomes

$\mathrm{Pe}=\frac{2}{\exp \left(n^{2} \sigma^{2}\right)-1}$

which suggests that the variance in the logarithm of the meanaperture distribution can be directly obtained from the Peclet number by

$\sigma^{2}=\frac{1}{n^{2}} \ln \left(1+\frac{2}{\mathrm{Pe}}\right)$

In field tracer tests, $\mathrm{Pe}$ is often found to lie in the range between 1 and 100 with a predominance of $\mathrm{Pe}=1 \sim 10$ (Gelhar 1993; Neretnieks 2002), which actually defines the pulse spreading (dispersion) by velocity dispersion (Neretnieks 1983). Velocity dispersion always gives a constant $\mathrm{Pe}$, as indicated by Eq. (81), and a dispersion coefficient $D_{\mathrm{f}} \propto x$ for a given variance of the aperture distribution, which also implies that the classical advection-dispersion equation cannot be used for the ensemble of flow paths when velocity dispersion is active.

Moreover, assuming the mean aperture, the mean halfwidth and the mean flow rate to be known entities, it can be obtained from Eqs. (75) and (76) that

$k_{m}=W_{\text {mean }} \exp \left(-\frac{2 m \mu+m^{2} \sigma^{2}}{2}\right)$

and

$k_{n}=\frac{q_{\text {mean }}}{2 C_{\mathrm{q}} W_{\text {mean }}} \exp \left[-\frac{2(n+1) \mu+(2 m+n+1)(n+1) \sigma^{2}}{2}\right]$

which allows for Eqs. (69), (71) and (72), respectively, to be rewritten as
$W=W_{\text {mean }} \exp \left(-\frac{2 m \mu+m^{2} \sigma^{2}}{2}\right) \bar{a}^{m}$

and

$q=q_{\text {mean }} \exp \left[-\frac{2(m+n+1) \mu+(m+n+1)^{2} \sigma^{2}}{2}\right] \bar{a}^{m+n+1}$

and

$u=\frac{q_{\text {mean }}}{2 W_{\text {mean }}} \exp \left[-\frac{2(n+1) \mu+(2 m+n+1)(n+1) \sigma^{2}}{2}\right] \bar{a}^{n}$

Apparently, the last two relations are independent of the channel geometry, which is correct if and only if all the channels have the same geometry. Imagine a fracture consisting of a rectangular channel and a tapered channel. The mean flow rate will be different than that of a fracture consisting of two rectangular channels. As a result, the $C_{\mathrm{q}}$ factor in Eq. (71) for the individual flow rate cannot be canceled out by the $C_{\mathrm{q}}$ factors present in Eq. (76) for the mean flow rate. This suggests that these results cannot be extended to general cases where the fracture or ensemble flow-path is composed of channels with different geometries. In addition, it should be stressed that, in deriving the above relations, the $C_{\mathrm{q}}$ factor is assumed constant, independent of the mean aperture $\bar{a}$. This is, nevertheless, the case also for channels with varying aperture, as shown in the subsequent.

Following this discussion, the authors now proceed to channels with varying aperture across the width. Exemplified for tapered channels, the local aperture $a$ decreases linearly along the channel width, as shown in Fig. 16. The mean aperture $\bar{a}$ is, then, one half of the largest aperture $a_{0}$ in each of the channels whereas the half-aperture $b$ is given by, for each of the channels,

$b=\frac{1}{1+\sqrt{1+\left(a_{0} / W\right)^{2}}} \frac{a_{0}}{2}$

This gives, by substituting Eq. (69) for $W$ and $a_{0}=2 \bar{a}$,

$b=\frac{2}{1+\sqrt{1+4 k_{m}^{-2} \bar{a}^{2-2 m}}} \frac{\bar{a}}{2}$

Therefore, the shape factor $C_{\mathrm{s}}$ in Eq. (70) becomes

$C_{\mathrm{s}}=\frac{2}{1+\sqrt{1+4 k_{m}^{-2} \bar{a}^{2-2 m}}}$ 
In addition, the volumetric flow rate is given by

$q=\frac{2}{n+2} W k_{n} a_{0}^{n+1}=\frac{2^{n+2}}{n+2} k_{m} k_{n} \bar{a}^{m+n+1}$

which allows for writing the factor $C_{\mathrm{q}}$ in Eq. (71) as

$C_{\mathrm{q}}=\frac{2^{n+1}}{n+2}$

In the case of sinusoidal wave-shaped channels, the local aperture $a$ decreases along the channel width in a way as described by Eq. (64). The mean aperture $\bar{a}$ is, then, also one half of the largest aperture $a_{0}$ in each of the channels. The halfaperture $b$, which determines the flow wetted surface-volume relationship, is however given for each of the channels by,

$$
b=\frac{1}{1+\frac{2}{\pi} \sqrt{1+\left(\frac{\pi a_{0}}{2 W}\right)^{2}} E\left(\left[1+\left(\frac{2 W}{\pi a_{0}}\right)^{2}\right]^{-1 / 2}\right)^{\frac{a_{0}}{2}}}
$$

where $E(k)$ is the complete elliptic integral of the second kind, taking values between 1.0 and $\pi / 2$.

Consequently, the shape factor $C_{\mathrm{s}}$ in Eq. (70) can be written as

$$
C_{\mathrm{S}}=\frac{2}{1+\frac{2}{\pi} \sqrt{1+\pi^{2} k_{m}^{-2} \bar{a}^{(2-2 m)}} E\left(\left[1+\pi^{-2} k_{m}^{2} \bar{a}^{(2 m-2)}\right]^{-1 / 2}\right)}
$$

Moreover, it can be found that the volumetric flow rate for each of the wave-shaped channels can generally be given by

$$
\begin{aligned}
q & =\frac{1}{2^{n}} \frac{W}{\pi} k_{n} a_{0}^{n+1} \int_{0}^{\pi}(1+\cos x)^{n+1} \mathrm{~d} x \\
& =\frac{2}{\pi} k_{m} k_{n} \bar{a}^{m+n+1} \int_{0}^{\pi}(1+\cos x)^{n+1} \mathrm{~d} x
\end{aligned}
$$

, indicating by comparing with Eq. (71), that the factor $C_{\mathrm{q}}$ now becomes

$C_{\mathrm{q}}=\frac{1}{\pi} \int_{0}^{\pi}(1+\cos x)^{n+1} \mathrm{~d} x$

which is 5.5 in the case of $n=2$.

Clearly, the $C_{\mathrm{q}}$ factors as given in Eqs. (92) and (96) only depend on the value of the $n$ parameter. As a result, Eqs. (86) and (87) would always hold regardless of the geometry of the channels as long as they are of the same shape. This also suggests that the joint $f$ function, as defined in Eq. (45), would be identical for two fractures consisting of rectangular and tapered channels, respectively, if assuming for now the dispersion coefficient $D_{\mathrm{f}}$ to have the same value.

The $C_{\mathrm{s}}$ factors as given in Eqs. (90) and (94) depend, however, apparently on the mean aperture $\bar{a}$ in cases of $m \neq 1$. It therefore seems that if one tries to evaluate the mean value of $b, b_{\text {mean }}$, the result would be rather complex. However, since $k_{m}$ is on the order of $W_{\text {mean }}$, it would be reasonable to take $C_{\mathrm{s}} \approx 1$ for all kind of channels. Alternatively, it can simply be evaluated by setting $k_{m}=W_{\text {mean }}$ and $\bar{a}=a_{\text {mean }}$ in Eqs. (90) and (94). This allows the following equation to be written with the help of Eq. (70):

$b_{\text {mean }}=\int_{0}^{\infty} b p(\bar{a}) \mathrm{d} \bar{a}=C_{\mathrm{s}}\left(\frac{a_{\text {mean }}}{2}\right)$

The averaged $g(t)$ function as given in Eq. (42), which describes the impact of the matrix diffusion, would then not be influenced much by the geometry of the channels. The geometry influences the Taylor-like dispersion, which, however, is accounted for by the f-function.

\section{Appendix D: Nomenclature}

a Local aperture of the channel (L)

$\bar{a} \quad$ Mean of the local apertures of the channels (L)

$a_{0} \quad$ Largest aperture of the tapered channel (L)

$a_{\text {mean }} \quad$ Mean aperture of the ensemble of channels (L)

$b \quad$ Half aperture of a channel with arbitrary geometry, FLV/FWS (L)

$b_{\text {mean }} \quad$ Mean aperture of the ensemble of channels (L)

$c_{\text {in }} \quad$ Concentration of the tracer at the origin of the fracture $\left(\mathrm{ML}^{-3}\right)$

c Tracer concentration $\left(\mathrm{ML}^{-3}\right)$

$c_{\mathrm{p}} \quad$ Pore-water concentration in the rock matrix $\left(\mathrm{ML}^{-3}\right)$

$C_{\mathrm{s}}, C_{\mathrm{q}} \quad$ Shape factors to consider the channel geometry effect on flow condition (-)

$D_{\mathrm{f}} \quad$ Longitudinal dispersion coefficient in the fracture or channel $\left(\mathrm{L}^{2} \mathrm{~T}^{-1}\right)$

$D_{\mathrm{p}} \quad$ Pore diffusion coefficient in the rock matrix $\left(\mathrm{L}^{2} \mathrm{~T}^{-1}\right)$

$D_{\mathrm{w}} \quad$ Molecular diffusion coefficient in water $\left(\mathrm{L}^{2} \mathrm{~T}^{-1}\right)$

FLV Flow volume in the single channel $\left(\mathrm{L}^{3}\right)$

FWS Area of flow-wetted surface of the single fracture or channel $\left(\mathrm{L}^{2}\right)$

$G \quad$ Diffusive mass-transfer parameter $\left(\mathrm{T}^{-1 / 2}\right)$

$H \quad$ Heaviside step function

$k_{m} \quad$ Proportionality constant that relates the channel half width to the mean of the local apertures of the channels, $\bar{a}\left(\mathrm{~L}^{(1-m)}\right)$

$k_{n} \quad$ Proportionality constant that relates the local water velocity to the local aperture in the channel $\left(\mathrm{L}^{-1} \mathrm{~T}^{-1}\right)$

$L \quad$ Channel length (L)

$m \quad$ Power law exponent that relates the channel half width to the mean of the local apertures of the channels, $\bar{a}(-)$

$m_{0} \quad$ Ratio between the mass injected instantaneously and the volumetric flow rate through the fracture $\left(\mathrm{MTL}^{-3}\right)$

MPG Material property group of the rock matrix $\left(\mathrm{LT}^{-1 / 2}\right)$ 
$n \quad$ Power law exponent that relates the local water velocity to the local aperture in the channel (-)

$N \quad$ Number of the independent channels that make up of the fracture $(-)$

Pe $\quad$ Peclet number $(-)$

$q_{\mathrm{f}} \quad$ Volumetric flow rate of a fictitious stream tube in the fracture $\left(\mathrm{L}^{3} \mathrm{~T}^{-1}\right)$

$q_{i} \quad$ Volumetric flow rate in the $i$ th channel $\left(\mathrm{L}^{3} \mathrm{~T}^{-1}\right)$

$q_{\text {mean }} \quad$ Mean flow rate of the ensemble of channels $\left(\mathrm{L}^{3} \mathrm{~T}^{-1}\right)$

$q_{\mathrm{w}} \quad$ Mean flow rate of the fictitious stream tubes $\left(\mathrm{L}^{3} \mathrm{~T}^{-1}\right)$

$Q \quad$ Volumetric flow rate through the fracture $\left(\mathrm{L}^{3} \mathrm{~T}^{-1}\right)$

$R_{\mathrm{f}} \quad$ Retardation coefficient in the fracture (-)

$R_{\mathrm{p}} \quad$ Retardation coefficient of the rock matrix (-)

$s \quad$ Laplace transform variable $\left(\mathrm{T}^{-1}\right)$

$t \quad$ Time (T)

$t_{\mathrm{w}} \quad$ Mean water residence time in the fracture or channel

(T)

$t_{\mathrm{w}, \text { mean }}$ Mean water residence time of the ensemble of channels (T)

$u \quad$ Mean water velocity in the fracture or channel $\left(\mathrm{LT}^{-1}\right)$

$u_{\mathrm{x}} \quad$ Local water velocity in the channel $\left(\mathrm{LT}^{-1}\right)$

$W \quad$ Half width of the channel (L)

$W_{\text {mean }} \quad$ Mean half-width of the ensemble of channels (L)

$x \quad$ Distance along the flow direction (L)

$z \quad$ Distance into the rock matrix (L)

$\delta \quad$ Dirac delta function

$\varepsilon \quad$ Porosity of the rock matrix (-)

$\lambda \quad$ Decay constant $\left(\mathrm{T}^{-1}\right)$

$\mu \quad$ Mean of the log-normal distribution of $\bar{a}(-)$

$\sigma \quad$ Standard deviation of the log-normal distribution of $\overline{a(-)}$

$\sigma_{\mathrm{t}} \quad$ Standard deviation of the water residence time (T)

$\tau \quad$ Solute residence time in the fracture in the absence of retarding mechanisms and $\lambda=0(\mathrm{~T})$

Open Access This article is distributed under the terms of the Creative Commons Attribution 4.0 International License (http:// creativecommons.org/licenses/by/4.0/), which permits unrestricted use, distribution, and reproduction in any medium, provided you give appropriate credit to the original author(s) and the source, provide a link to the Creative Commons license, and indicate if changes were made.

\section{References}

Becker MW, Shapiro AM (2000) Tracer transport in fractured crystalline rock: evidence of nondiffusive breakthrough tailing. Water Resour Res 36(7):1677-1686

Becker MW, Shapiro AM (2003) Interpreting tracer breakthrough tailing from different forced-gradient tracer experiment configurations in fractured bedrock. Water Resour Res 39(1)

Benson DA, Wheatcrat SW, Meerschaert MM (2000) Application of a fractional advection-dispersion equation. Water Resour Res 36: $1403-1421$
Berkowitz B (2002) Characterizing flow and transport in fractured geological media: a review. Adv Water Resour 25:861-884

Berkowitz B, Bear J, Braester C (1988) Continuum models for contaminant transport in fractured porous formations. Water Resour Res 24(8):1225-1236

Berkowitz B, Cortis A, Dentz M, Scher H (2006) Modeling non-Fickian transport in geological formations as a continuous time random walk. Rev Geophys 44:RG2003

Bird RB, Stewart WE, Lightfoot EN (2002) Transport phenomena, 2nd edn. Wiley, New York

Bodin J (2015) From analytical solutions of solute transport equations to multidimensional time-domain random walk (TDRW) algorithms. Water Resour Res 51(3):1860-1871

Chesnut DA (1994) Dispersivity in heterogeneous media, Lawrence Livermore National Laboratory, UCRL-JC-114790, LLNL, Livermore, CA

Cirpka OA, Kitanidis PK (2000) An advective-dispersive stream tube approach for the transfer of conservative tracer data to reactive transport. Water Resour Res 36:1209-1220

Crawford J, Moreno L, Neretnieks I (2003) Determination of the flowwetted surface in fractured media. J Cont Hydrol 61(1-4):361-369

De Hoog FR, Knight JH, Stokes AN (1982) An improved method for numerical inversion of Laplace transforms. SIAM J Sci Stat Comput 3(3):357-366

Gelhar LW (1993) Stochastic subsurface hydrology. Prentice Hall, New York

Gelhar LW, Welty C, Rehfeldt KR (1992) A critical review of data on field-scale dispersion in aquifers. Water Resour Res 28(7):19551974

Ginn TR (2001) Stochastic-convective transport with nonlinear reactions and mixing: finite streamtube ensemble formulation for multicomponent reaction systems with intra-streamtube dispersion. J Cont Hydrol 47(1):1-28

Gylling B, Moreno L, Neretnieks I (1999) The channel network model: a tool for transport simulation in fractured media. Ground Water 37 : $367-375$

Haggerty R (1999) Application of the multirate diffusion approach in tracer test studies at Äspö HRL, SKB Rep. R-99-62, Swedish Nuclear Fuel and Waste Management Company, Stockholm

Haggerty R, Gorelick SM (1995) Multiple-rate mass transfer for modeling diffusion and surface reactions in media with pore-scale heterogeneity. Water Resour Res 31(10):2383-2400

Hakami E, Larsson E (1996) Aperture measurements and flow experiments on a single natural fracture. Int J Rock Mech Min Sci Geomech Abstr 33(4):395-404

Hjerne C, Nordqvist R, Harrström J (2010) Compilation and analyses of results from cross-hole tracer tests with conservative tracers, SKB Rep. R-09-28, Swedish Nuclear Fuel and Waste Management Company, Stockholm

Hodgkinson DP, Maul PR (1988) 1-D modelling of radionuclide migration through permeable and fractured rock for arbitrary length decay chain using numerical inversion of Laplace transforms. Ann Nucl Energy 15(4):175-189

Keller AA (1997) High resolution CAT imaging of fractures in consolidated materials. Int J Rock Mech Min Sci 34(3/4):358-375

Liu L, Neretnieks I (2005) Analysis of fluid flow and solute transport in a fracture intersecting a canister with variable aperture fractures and arbitrary intersection angles. Nucl Technol 150:132-144

Liu L, Neretnieks I (2006) Analysis of fluid flow and solute transport through a single fracture with variable apertures intersecting a canister: comparison between fractal and Gaussian fractures. Phys Chem Earth 31:634-639

Long JCS, Remer JS, Wilson CR, Witherspoon PA (1982) Porous media equivalents for networks of discontinuous fractures. Water Resour Res 18(3):645-658 
Mahmoudzadeh B, Liu L, Moreno L, Neretnieks I (2013) Solute transport in fractured rocks with stagnant water zone and rock matrix composed of different geological layers: model development and simulations. Water Resour Res 49:1-19. doi:10.1002/wrcr.20132

Mahmoudzadeh B, Liu L, Moreno L, Neretnieks I (2016) Solute transport through fractured rock: radial diffusion into the rock matrix with several geological layers for an arbitrary length decay chain. J Hydrol 536:133-146

Matheron G, De Marsily G (1980) Is transport in porous media always diffusive? A counterexample. Water Resour Res 16(5):901-917

McKay LD, Gillham RW, Cherry JA (1993) Field experiments in a fractured clay till: 2 . solute and colloid transport. Water Resour Res 29(12):3879-3890

Moreno L, Tsang CF (1994) Flow channeling in strongly heterogeneous porous media: a numerical study. Water Resour Res 30:1421-1421

Moreno L, Tsang YW, Tsang CF, Hale FV, Neretnieks I (1988) Flow and tracer transport in a single fracture: a stochastic model and its relation to some field observations. Water Resour Res 24(12):20332048

Neretnieks I (1980) Diffusion in the rock matrix: an important factor in radionuclide retardation? J Geophys Res 85(B8):4379-4397

Neretnieks I (1983) A note on fracture flow dispersion mechanisms in the ground. Water Resour Res 19(2):364-370

Neretnieks I (2002) A stochastic multi-channel model for solute transport: analysis of tracer tests in fractured rock. J Cont Hydrol 55:175-211

Neretnieks I, Moreno L (2003) Prediction of some in situ tracer tests with sorbing tracers using independent data. J Cont Hydrol 62(1-4):351360

Rasmuson A, Neretnieks I (1986) Radionuclide transport in fast channels in crystalline rock. Water Resour Res 22(8):1247-1256
Shahkarami P, Liu L, Moreno L, Neretnieks I (2015) Radionuclide migration through fractured rock for arbitrary-length decay chain: analytical solution and global sensitivity analysis. J Hydrol 520:448460

Shahkarami P, Liu L, Moreno L, Neretnieks I (2016) The effect of stagnant water zones on retarding radionuclide transport in fractured rocks: an extension to the channel network model. J Hydrol 540: $1122-1135$

Simmons CS, Ginn TR, Wood BD (1995) Stochastic-convective transport with nonlinear reaction: mathematical framework. Water Resour Res 31(11):2675-2688

Šimůnek J, van Genuchten MT (2008) Modeling nonequilibrium flow and transport processes using HYDRUS. Vadose Zone J 7(2):782-797

Šimůnek J, Jarvis NJ, Van Genuchten MT, Gärdenäs A (2003) Review and comparison of models for describing non-equilibrium and preferential flow and transport in the vadose zone. J Hydrol 272(1):14 35

Sudicky EA, Frind EO (1982) Contaminant transport in fractured porous media: analytical solutions for a system of parallel fractures. Water Resour Res 18(6):1634-1642

Tang DH, Frind EO, Sudicky EA (1981) Contaminant transport in fractured porous media: analytical solution for a single fracture. Water Resour Res 17(3):555-564

Taylor G (1953) Dispersion of soluble matter in solvent flowing slowly through a tube. Proc R Soc Lond Ser A 219(1137):186-203

Tsang C-F, Neretnieks I (1998) Flow channeling in heterogeneous fractured rocks. Rev Geophys 36(2):275-298

Wang L, Cardenas MB, Deng W, Bennett PC (2012) Theory for dynamic longitudinal dispersion in fractures and rivers with Poiseuille flow. Geophys Res Lett 39(5):L05401. doi:10.1029/2011g1050831 